\title{
Locally Noncommutative Space-Times
}

\author{
Dorothea Bahns* and Stefan Waldmann ${ }^{\S}$
}

July 2006

FR-THEP 2006/12, ZMP-HH/2006-13

\begin{abstract}
Localized noncommutative structures for manifolds with connection are constructed based on the use of vertical star products. The model's main feature is that two points that are far away from each other will not be subject to a deviation from classical geometry while space-time becomes noncommutative for pairs of points that are close to one another.
\end{abstract}

\section{Introduction}

Models of noncommutative space-times have become increasingly popular in the recent past and are believed to be reasonable candidates for Planck scale physics, at least as an intermediate step towards a full quantum mechanical treatment of geometry. The main idea is that in the regime where quantum theory and general relativity are no longer independent, the notion of a point in space-time becomes meaningless, and a finite minimal length or uncertainty relations for coordinate functions have to be postulated in order to prohibit the localization of points with arbitrarily high precision. Models with uncertainty relations are usually implemented by considering noncommuting coordinate operators, replacing the coordinate functions.

More concretely, the situation can be seen as follows: for the noncommutative algebra describing the noncommutative space-time one chooses a symbol calculus resulting in a Poisson structure $\theta$ on the classical space-time together with a corresponding quantization of some reasonable class of functions on $M$. Quantization is achieved either analytically, see e.g. [15,28], or in the context of deformation quantization [3] in form of a star product, see e.g. [21]. A particularly simple and wellstudied example is the noncommutative Minkowski space $M=\mathbb{R}^{1,3}$ where the Poisson structure is chosen to be constant and symplectic. Of course, this is a highly non-geometric situation and in view of an aim towards general relativity, this model has to be understood as a toy-model that should be surpassed by a more geometric approach.

Having established a noncommutative space-time, it is of particular interest to study dynamics in it, usually in form of a quantum field theory defined on such a space-time; and much work has been done in that direction. However, various technical but more importantly, also conceptual problems occur in this case, one of which we want to address here: the global nature of the Poisson structure, in particular in the case of constant $\theta$ on Minkowski space-time, necessarily results in effects that are visible at large distances.

\footnotetext{
*Department für Mathematik, Universität Hamburg, Bundesstr. 55, D-20253 Hamburg, Germany, bahns@math . uni-hamburg. de

${ }^{\S}$ Fakultät für Mathematik und Physik, Albert-Ludwigs-Universität Freiburg, Physikalisches Institut, HermannHerder-Straße 3, D 79104 Freiburg, Germany, Stefan.Waldmann@physik.uni-freiburg.de
} 
In quantum mechanics, this situation occurs in the sense that large momenta certainly play a crucial role in quantum effects, i.e. quantum mechanics is very well observable at large distances in phase space. However, there is a polarization separating momenta and coordinates from one another and their respective 'magnitudes' are comparable only after a suitable scaling involving characteristic parameters of the system as well as Planck's constant $\hbar$. Clearly, with regard to the commutation relations, the case of constant nondegenerate $\theta$ in noncommutative Minkowski space is mathematically equivalent to quantum mechanics in a space of half the dimension. In this case, however, all noncommuting operators are of the same kind, and short distance/long distance effects are expected to mix.

In any case, more sophisticated investigations seem to indicate that a constant $\theta$ leads to macroscopic effects that (if the theory's predictions are taken seriously) would have to be visible. A prominent example is the mixing of ultraviolet and infrared divergences that appears in Euclidean noncommutative field theories. A related effect is the modification of the dispersion relation in field theories with Lorentz signature, where the largest deviation from the ordinary relation appears at small momenta, see e.g. [24].

Moreover, a violation of microcausality at all scales of distances results from the application of the highly nonlocal twisted convolution product (i.e. the Weyl-Moyal product in its integral form), see however the discussion of the cluster decomposition property in [1]. It should be noted that for Lie-type Poisson structures, i.e. linear Poisson structures on Minkowski space-time, or quadratic Poisson structures as arising from a quantum group approach, this situation is even worse.

This leads to the following natural question: can we modify the noncommutativity in such a way that it decays in a reasonable way for large distances? Considering a non-constant $\theta$ that vanishes in (spatial) infinity does of course not solve the problem: in such a scenario, the noncommutativity would vary within the universe, such that some regions would have 'more' or 'less' noncommutativity than others, depending on the absolute position.

Instead, our suggestion is to take the concept of distance as a starting point and consider the distance of two points. Hence, in our approach, $M \times M$ is endowed with a noncommutative structure, instead of $M$ itself alone. It is now straightforward to define noncommutativity only at small distances: we simply consider a Poisson structure and a corresponding star product on $M \times M$ that is nontrivial only close to the diagonal $\Delta_{M}$ in $M \times M$ and zero or quickly decreasing away from $\Delta_{M}$.

The aim of this paper now is to set up a kinematical framework for such types of noncommutativity and explore some of their features. The whole approach has to be understood as a first step as we have not yet investigated any form of dynamics on our locally noncommutative space-time. This will be subject to future projects.

Our paper is organized as follows: In Section 2 we first discuss the general framework of star products on $M \times M$ localized close to the diagonal and use the exponential map of the Levi-Cività connection of the space-time manifold $M$ to pull-back everything to the tangent bundle $T M$ of $M$. As crucial condition the verticality of the Poisson structure and the star product is discussed in detail. In Section 3 we investigate further properties of the vertical star product and show how they can be used to endow every point of $M$ with a small noncommutative neighborhood. Then we discuss in detail states and the corresponding expectation values of our observables including in particular the measurement of the (Lorentz) metric on $M$ itself. Section 4 is devoted to the particular example of flat Minkowski space-time. Though our approach is fully geometric in general, this provides an important and simple example which we investigate in detail. The last Section 5 contains a discussion of further open questions and possible extensions and limitations of the model. In Appendix A we have included a detailed technical discussion of vertical Poisson structures, vertical star products and the vertical formality theorem governing their existence and classification. 
Acknowledgement: We would like to thank Klaus Fredenhagen, Jakob Heller, Stefan Weiß, Julius Wess and Jochen Zahn for valuable discussions and remarks.

\section{Noncommutative Structure at Small Distances}

Starting point of our construction is a smooth $n$-dimensional manifold $M$ which allows for a Lorentz metric $g$. Note however, that our construction only depends on the respective Levi-Cività connection, such that we may (and frequently will) consider Riemannian metrics as well. Our aim is to define a noncommutative structure on $M$ whose effects are visible only at small distances in the sense that a pair of points $q, q^{\prime} \in M$ will 'feel' noncommutative effects only when they are in the close vicinity of the diagonal $\Delta_{M}$ in $M \times M$.

\subsection{Classical space-time prerequisites}

Let us first recall some well-known constructions from differential geometry we shall need later on. The Levi-Cività connection $\nabla$ of $g$ determines the geodesic structure of $M$ and thereby the exponential map exp, which is defined on some open neighborhood of the zero section of the tangent bundle $\pi: T M \longrightarrow M$. We choose once and for all such an open neighborhood $\mathcal{U} \subseteq T M$ of the zero section with the property that the map

$$
\Phi: T M \supseteq \mathcal{U} \ni v_{p} \mapsto \Phi\left(v_{p}\right)=\left(\exp _{p}\left(-v_{p}\right), \exp _{p}\left(v_{p}\right)\right) \in M \times M \quad \text { where } \quad v_{p} \in T_{p} M,
$$

is a diffeomorphism onto its image $\mathcal{V} \subseteq M \times M$. Here, $v_{p} \in T_{p} M$ denotes a tangent vector at $p \in M$ and $\exp _{p}$ is the exponential map at $p$, i.e. $t \mapsto \exp _{p}\left(t v_{p}\right)$ is the geodesic through $p$ at $t=0$ with initial velocity $v_{p}$. Clearly, $\Phi$ maps the zero section diffeomorphically to the diagonal $\Delta_{M} \subseteq M \times M$ and $\mathcal{V}$ is an open neighborhood of $\Delta_{M}$.

For $p \in M$ we set $\mathcal{U}_{p}=\mathcal{U} \cap T_{p} M$ which is an open neighborhood of $0_{p} \in T_{p} M$. Its image $\mathcal{V}_{p}=\exp _{p}\left(\mathcal{U}_{p}\right)$ is an open neighborhood of $p \in M$. As usual, we obtain the well-known normal coordinates $x^{1}, \ldots, x^{n}: \mathcal{V}_{p} \longrightarrow \mathbb{R}$ on $\mathcal{V}_{p}$ by choosing linear coordinates $\xi^{1}, \ldots, \xi^{n}$ with respect to some vector space basis $e_{1}, \ldots, e_{n}$ on $T_{p} M$ and setting $x^{i}=\xi^{i} \circ \exp _{p}^{-1}$. As $T_{p} M$ is equipped with the metric $g_{p}$ we can even choose $e_{1}, \ldots, e_{n}$ to be orthonormal.

The geometric interpretation of $\Phi$ is now the following: suppose $\left(q, q^{\prime}\right) \in \mathcal{V} \subseteq M \times M$ are within the image of $\Phi$. Then $\Phi^{-1}\left(q, q^{\prime}\right)=v_{p} \in \mathcal{U}_{p} \subseteq T_{p} M$ and $v_{p}$ is such that $\exp _{p}\left(-v_{p}\right)=q$ and $\exp _{p}\left(v_{p}\right)=q^{\prime}$. Thus the point $p$ is the geodesic midpoint between $q$ and $q^{\prime}$. The normal coordinates around $p$ can be seen as the geodesic relative coordinates of $\left(q, q^{\prime}\right)$ with respect to their 'center of mass' $p$. This explains our definition of $\Phi$ since this way the situation for $q$ and $q^{\prime}$ becomes most symmetric: we denote by $\tau_{M \times M}: M \times M \longrightarrow M \times M$ the global diffeomorphism $\tau_{M \times M}\left(q, q^{\prime}\right)=\left(q^{\prime}, q\right)$ and by $\tau_{T M}: T M \longrightarrow T M$ the global diffeomorphism $\tau_{T M}\left(v_{p}\right)=-v_{p}$. Then we have

$$
\Phi \circ \tau_{T M}=\tau_{M \times M} \circ \Phi
$$

on $\mathcal{U} \cap \tau_{T M}(\mathcal{U})$. Thus it is natural to demand that $\mathcal{U}$ is invariant under the reflection $\tau_{T M}$. We will always assume that this is the case. As a consequence also $\mathcal{V}$ is symmetric under the exchange $\tau_{M \times M}$.

\subsection{Noncommutativity at small distances}

We will now give a definition of a noncommutative structure that is nontrivial only in a vicinity of the diagonal $\Delta_{M}$ in $M \times M$. For technical reasons, we will use formal star products, i.e. we equip the 


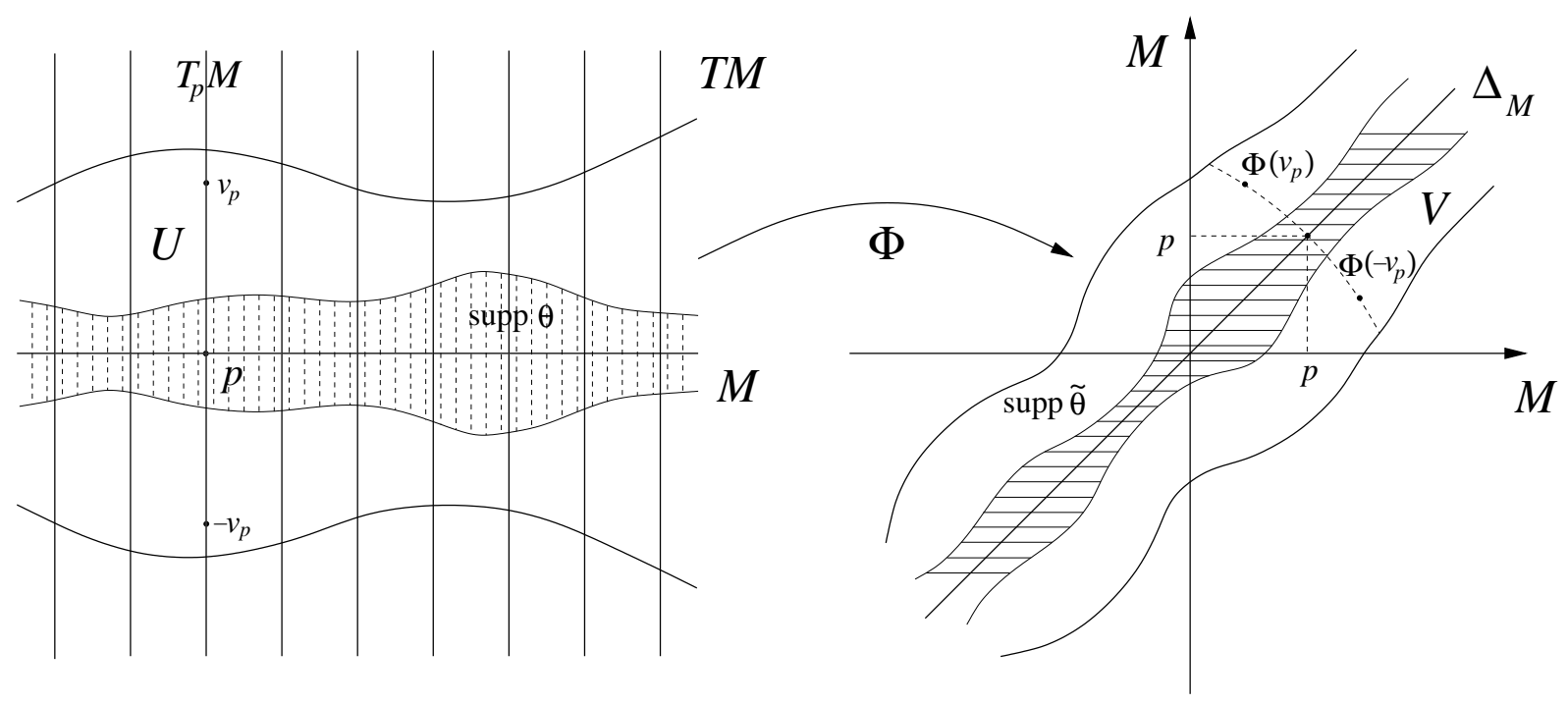

Figure 1: The exponential map $\Phi$ transports $\theta$ from $T M$ to $\tilde{\theta}$ on $M \times M$.

algebra of functions $C^{\infty}(M \times M)$ (which serve as the theory's observables) with a noncommutative product given by a star product $\tilde{\star}$.

Recall that a star product [3] on a manifold $N$ is a formal $\mathbb{C}[[\lambda]]$-bilinear associative deformation $\star$ of the algebra of smooth functions $C^{\infty}(N)$ written as

$$
f \star g=\sum_{r=0}^{\infty} \lambda^{r} C_{r}(f, g),
$$

where $f, g \in C^{\infty}(N)[[\lambda]]$, the $C_{r}$ are bidifferential operators such that $\star$ is associative and $1 \star f=f=$ $f \star 1$ for all $f$. Additionally, we want $\star$ to be a deformation of the ordinary product of functions in the sense that $C_{0}(f, g)=f g$ is the undeformed commutative product. As a consequence of associativity, $\{f, g\}=\frac{1}{\mathrm{i}}\left(C_{1}(f, g)-C_{1}(g, f)\right)$ defines a Poisson bracket and thus a Poisson bivector field $\theta \in \Gamma^{\infty}\left(\Lambda^{2} T N\right)$ by $\{f, g\}=\langle\theta, \mathrm{d} f \otimes \mathrm{d} g\rangle$. The Jacobi identity for $\{\cdot, \cdot\}$ is equivalent to $\llbracket \theta, \theta \rrbracket=0$ where $\llbracket \cdot, \cdot \rrbracket$ denotes the Schouten-Nijenhuis bracket of multivector fields. Recent reviews on deformation quantization can be found in [13,18]. For an elementary introduction see [33].

In our case, we consider a star product $\tilde{\star}$ on $M \times M$ whose first order term yields a Poisson bivector $\tilde{\theta} \in \Gamma^{\infty}(T(M \times M))$ on $M \times M$ (conversely, any such Poisson bivector can be 'quantized' into a star product $\tilde{\star})$. The deformation parameter $\lambda$ is a formal parameter, but may be thought of as a Planck area in our context. Our crucial requirement now is that the support of $\tilde{\theta}$ be close to the diagonal $\Delta_{M} \subset M \times M$, such that the product of functions differs from the pointwise one only at small distances.

First, we require that $\operatorname{supp} \tilde{\theta} \subseteq \mathcal{V} \subseteq M \times M$. This allows to pull-back $\tilde{\theta}$ via the diffeomorphism $\Phi$ in order to obtain a Poisson bivector $\theta=\Phi^{*} \tilde{\theta} \in \Gamma^{\infty}\left(\Lambda^{2} T \mathcal{U}\right)$. Since $\operatorname{supp} \tilde{\theta} \subseteq \mathcal{V} \subseteq M \times M$, the Poisson bivector $\theta$ extends to a globally defined Poisson bivector on $T M$ with support $\operatorname{supp} \theta \subseteq \mathcal{U}$, see also Figure 1. Secondly, we require that $\operatorname{supp} \theta \cap T_{p} M$ is compact in $T_{p} M$. This expresses in a purely topological manner that the support of $\theta$ is 'small': instead of using the (Lorentz) metric explicitly, we simply fix the 'range of noncommutativity' (given by the support of $\theta$, or $\tilde{\theta}$ respectively) to remain finite.

The last requirement is to admit only such Poisson bivectors $\tilde{\theta}$ as are invariant under $\tau_{M \times M}$. 


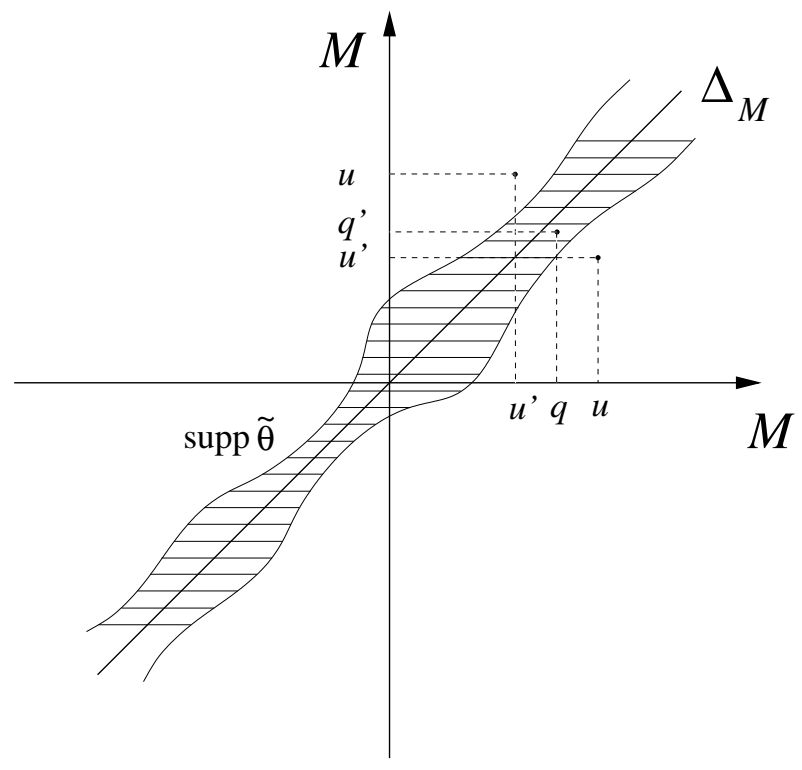

Figure 2: Some pairs of points are within the range of noncommutativity, some are not. Here the support of $\tilde{\theta}$ is symmetric under the canonical flip $\tau$.

By (2.2), this ensures invariance of $\theta$ under $\tau_{T M}$, such that a pair of points $\left(q, q^{\prime}\right)$ is within the range of noncommutativity if and only if $\left(q^{\prime}, q\right)$ is, see Figure 2.

Starting from such a Poisson bivector $\tilde{\theta}$ as first order term in the star product, the construction methods for star products like those in $[12,14,16,22]$ will yield star products $\tilde{\star}$, whose higher order bidifferential operators $\tilde{C}_{r}$ for $r \geq 1$ still have support contained in supp $\tilde{\theta}$. In principle, there exist more general star products not obeying this support condition but we shall only use star product with $\operatorname{supp} \tilde{C}_{r} \subseteq \operatorname{supp} \tilde{\theta}$. Clearly, such star products will reduce to the ordinary pointwise product of functions outside the support of $\tilde{\theta}$,

$$
\left.(f \tilde{\star} g)\right|_{M \times M \backslash \operatorname{supp} \tilde{\theta}}=\left.(f g)\right|_{M \times M \backslash \operatorname{supp} \tilde{\theta}}
$$

for all $f, g \in C^{\infty}(M \times M)[[\lambda]]$. Finally, one can easily arrange that the symmetry $\tau_{M \times M}$ remains a symmetry for $\tilde{\star}$, i.e. that

$$
\tau_{M \times M}^{*}(f \tilde{\star} g)=\left(\tau_{M \times M}^{*} f\right) \tilde{\star}\left(\tau_{M \times M}^{*} g\right)
$$

for all $f, g \in C^{\infty}(M \times M)[[\lambda]]$. In the following we shall always assume that $\tilde{\star}$ meets all these requirements. In fact, we shall discuss even more particular star products and give a concrete construction for them later.

Thanks to the support properties of $\tilde{\star}$ we can pull back each bidifferential operator $\tilde{C}_{r}$ to a bidifferential operator $C_{r}$ on $\mathcal{U} \subseteq T M$ via the diffeomorphism $\Phi: \mathcal{U} \longrightarrow \mathcal{V}$. Then these bidifferential operators yield a star product $\star$ on $\mathcal{U}$ with first order term corresponding to $\theta$. Thanks to $\operatorname{supp} C_{r} \subseteq$ $\operatorname{supp} \theta \subseteq \mathcal{U}$ for all $r \geq 1$, the star product $\star$ extends to $T M$. Conversely, any star product $\star$ for $\theta$ with the property $\operatorname{supp} C_{r} \subseteq \operatorname{supp} \theta \subseteq \mathcal{U}$ for all $r \geq 1$ can be pushed forward via $\Phi$ to give a star product $\tilde{\star}$ on $\mathcal{V}$ which extends to $M \times M$. Thus both points of view are entirely equivalent as long as we impose the support conditions. In particular, for $f, g \in C^{\infty}(M \times M)[[\lambda]]$ with $\operatorname{supp} f, \operatorname{supp} g \subseteq \mathcal{V}$ we have

$$
\Phi^{*}(f \tilde{\star} g)=\Phi^{*} f \star \Phi^{*} g .
$$




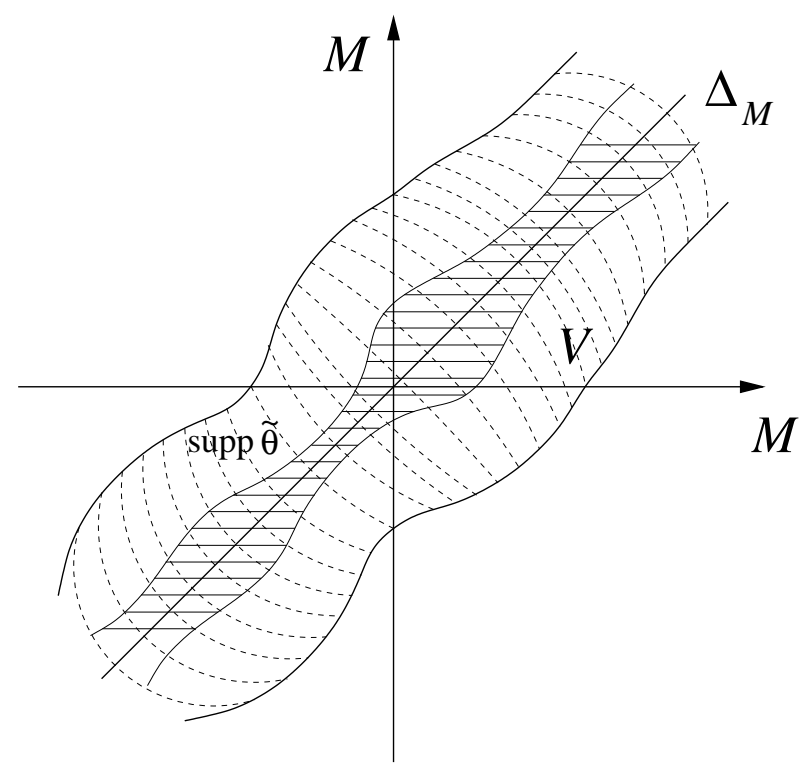

Figure 3: The dashed lines indicate the locally defined geodesic 'relative coordinates' transversal to the diagonal. They are only defined within $\mathcal{V}$ using the local diffeomorphism $\Phi$.

The property (2.5) translates into the symmetry

$$
\tau_{T M}^{*}(f \star g)=\tau_{T M}^{*} f \star \tau_{T M}^{*} g
$$

for all $f, g \in C^{\infty}(T M)[[\lambda]]$. In the following we shall use both descriptions and pass from one to the other freely.

\section{$2.3 \quad$ Vertical star products}

Up to now, the bivectors $\theta$ and $\tilde{\theta}$ as well as the corresponding star products $\star$ and $\tilde{\star}$, respectively, can still be very general as the support conditions alone are not very restrictive.

We now impose one further condition which implements the idea that it is only the distance between two points that determines whether noncommutativity is present, while their absolute position in space-time should not matter (though their absolute position may influence the specific form of noncommutativity via the bivector's parametric dependence on $p$ ). There being no intrinsic coordinates on $M \times M$ transversal to $\Delta_{M}$, we first use the map $\Phi$ to define geodesic relative coordinates near $\Delta_{M}$, see also Figure 3 . We now ask $\tilde{\star}$ to meet the following additional property: if after restriction to the open subset $\mathcal{V}$, a function $f \in C^{\infty}(M \times M)[[\lambda]]$ is constant with respect to the relative coordinates, then for any other function $g \in C^{\infty}(M \times M)[[\lambda]]$ we require

$$
f \tilde{\star} g=f g=g \tilde{\star} f .
$$

Note that (2.8) is trivially fulfilled outside of $\operatorname{supp} \tilde{\theta}$ by (2.4). In more physical terms, observables not sensitive to the relative coordinates should behave entirely classical, i.e. commutative. This makes our idea more precise, that $M \cong \Delta_{M} \subseteq M \times M$ should remain commutative as we have argued in the introduction: Indeed, functions $f \in C^{\infty}(M)[[\lambda]]$ can be prolongated at least locally on $\mathcal{V}$ from $M \cong \Delta_{M}$ to functions on $M \times M$ by defining them to be constant along the relative coordinates. Thus a non-trivial star product between such functions would result in a non-trivial multiplication law for functions on $M$. 
Again, we can translate (2.8) back to an equivalent statement regarding $\star$ on $T M$. Here, if $f \in C^{\infty}(T M)[[\lambda]]$ is constant along the fibers, i.e. of the form $f=\pi^{*} u$ with some $u \in C^{\infty}(M)[[\lambda]]$, then for any other $g \in C^{\infty}(T M)[[\lambda]]$ the star product becomes trivial,

$$
g \star \pi^{*} u=g \pi^{*} u=\pi^{*} u \star g .
$$

A more direct characterization of star products with this additional property is provided by the following theorem:

Theorem 2.1 A star product $\star$ on TM satisfies (2.9) if and only if $\star$ is vertical, i.e. each bidifferential operator $C_{r}$ differentiates only in vertical directions.

Note that for the non-trivial direction of this statement we have to use the associativity of $\star$. In the Appendix $\mathrm{A}$ we have collected informations on vertical Poisson structures and vertical star products including their existence and classification by means of a vertical formality theorem. As Theorem A.13 provides us a functorial construction of $\star$ out of a given $\theta$ guaranteeing all our requirements, the reader not interested in the technical details may safely proceed from here on. We summarize the data and requirements of our model in Table 1

\begin{tabular}{|c|c|}
\hline Semiclassical $\theta \in \Gamma^{\infty}\left(\Lambda^{2} T(T M)\right)$ & Formal deformation $\star=\sum_{r=0}^{\infty} \lambda^{r} C_{r}$ \\
\hline$\llbracket \theta, \theta \rrbracket=0$ (Jacobi identity) & $\star$ associative formal star product \\
$\theta$ vertical & $\star$ vertical \\
$\operatorname{supp} \theta \subseteq \mathcal{U}$ & $\operatorname{supp} C_{r} \subseteq \operatorname{supp} C_{1} \subseteq \mathcal{U}$ for all $r \geq 1$. \\
$\tau_{T M}^{*} \theta=\theta$ & $\tau_{T M}^{*}$ is automorphism of $\star$ \\
$\operatorname{supp} \theta \cap T_{p} M$ compact for all $p \in M$ & $\operatorname{supp} C_{r} \cap T_{p} M$ compact for all $p \in M$ \\
\hline
\end{tabular}

Table 1: Summary of the model

To make ourselves familiar with vertical star products, let us now give some local formulas. Let $\left(x^{1}, \ldots, x^{n}\right)$ be local coordinates on $U \subseteq M$ and denote by $\left(q^{1}=x^{1} \circ \pi, \ldots, q^{n}=x^{n} \circ \pi, v^{1}, \ldots, v^{n}\right)$ the induced coordinates on $T U \subseteq T M$. Here, as usual, a tangent vector $v_{p} \in T_{p} M$ is written as $v_{p}=v^{i}\left(v_{p}\right) \frac{\partial}{\partial x^{i}}$, thus specifying the linear coordinates $\left(v^{1}, \ldots, v^{n}\right)$ on the fibers. Now, a bivector $\theta \in \Gamma^{\infty}\left(\Lambda^{2} T(T M)\right)$ is vertical if and only if locally

$$
\left.\theta\right|_{T U}=\frac{1}{2} \theta^{i j} \frac{\partial}{\partial v^{i}} \wedge \frac{\partial}{\partial v^{j}}
$$

where $\theta^{i j} \in C^{\infty}(T U)$ are local coefficient functions depending on all variables, $q$ 's as well as $v$ 's. The condition $\tau_{T M}^{*} \theta=\theta$ means that the functions $\theta^{i j}$ must be even functions of the $v$ 's. The support condition is equivalent to $\theta^{i j}(q, \cdot)$ having compact support with respect to the $v$-coordinates for fixed $q^{1}, \ldots, q^{n}$. It follows directly from the definition that the star product $\star$ is vertical if and only if locally the bidifferential operators $C_{r}$ are of the form

$$
\left.C_{r}(f, g)\right|_{T U}=\sum_{I, J} C_{r}^{I J} \frac{\partial^{|I|} f}{\partial v^{I}} \frac{\partial^{|J|} g}{\partial v^{J}},
$$

with multi-indices $I$ and $J$, and where the local coefficient functions $C_{r}^{I J} \in C^{\infty}(T U)$ may again depend on $q$ 's as well as $v$ 's. The important point is that both functions are differentiated only in 
direction of the fiber variables. Of course, the $C_{r}^{I J}$ are subject to further conditions arising from the associativity of $\star$.

Local expressions for $\tilde{\theta}$ and $\tilde{\star}$ are more complicated as they require knowledge of the explicit form of the exponential map. This is only in very limited cases accessible whence we shall mainly work with $T M$ instead of $M \times M$. Note however, that for the interpretation of functions $f \in C^{\infty}(T M)$ as observables one should rather consider their counterparts on $M \times M$.

\section{$3 \quad$ Further properties of vertical star products}

Consider now a vertical Poisson structure $\theta$ and a corresponding star product $\star$ on $T M$ obeying the support conditions as well as the reflection symmetry $\tau_{T M}^{*} \theta=\theta$ and (2.7), respectively. Let

$$
\iota_{p}: T_{p} M \longrightarrow T M
$$

denote the embedding of the tangent space at $p \in M$ into the tangent bundle. As discussed in Appendix A.1 and Theorem A.13 we can restrict $\theta$ and $\star$ to a Poisson structure $\theta_{p}$ with corresponding Poisson bracket $\{\cdot, \cdot\}_{p}$ and a star product $\star_{p}$ on $T_{p} M$,

$$
\iota_{p}^{*}(\{f, g\})=\left\{\iota_{p}^{*} f, \iota_{p}^{*} g\right\}_{p}
$$

and

$$
\iota_{p}^{*}(f \star g)=\iota_{p}^{*} f \star_{p} \iota_{p}^{*} g
$$

for all $f, g \in C^{\infty}(T M)[[\lambda]]$. Here, it is important that the Poisson structure $\theta$ as well as the star product $\star$ are vertical, i.e. that all derivatives are only in the direction of the fibers. By construction, both structures are non-trivial only on $\mathcal{U}_{p}$.

In a last step we can push forward both $\theta_{p}$ and $\star_{p}$ to $M$ via the exponential map $\exp _{p}$. Since $\operatorname{supp} \theta_{p} \subseteq \mathcal{U}_{p}$ and $\exp _{p}$ is a diffeomorphism $\exp _{p}: \mathcal{U}_{p} \longrightarrow \exp _{p}\left(\mathcal{U}_{p}\right)=\mathcal{V}_{p}$ by our choice of $\mathcal{U}$, this is well-defined and yields a Poisson bivector $\tilde{\theta}_{p} \in \Gamma^{\infty}\left(\Lambda^{2} T \mathcal{V}_{p}\right)$. Again, the support conditions enable us to extend $\tilde{\theta}_{p}$ to all of $M$, whence we obtain a Poisson bivector $\tilde{\theta}_{p} \in \Gamma^{\infty}\left(\Lambda^{2} T M\right)$. Analogously, we obtain a star product $\tilde{\star}_{p}$ on $M$ which quantizes $\tilde{\theta}_{p}$. Now, by the very construction of $\tilde{\theta}_{p}$ and $\tilde{\star}_{p}$, for $f, g \in C^{\infty}(M)[[\lambda]]$ with $\operatorname{supp} f, \operatorname{supp} g \subseteq \mathcal{V}_{p}$,

$$
\left\{\exp _{p}^{*} f, \exp _{p}^{*} g\right\}_{\theta_{p}}=\exp _{p}^{*}\{f, g\}_{\tilde{\theta}_{p}}
$$

and

$$
\exp _{p}^{*} f \star_{p} \exp _{p}^{*} g=\exp _{p}^{*}\left(f \tilde{\star}_{p} g\right) .
$$

This way, every point $p \in M$ obtains its own star product $\tilde{\star}_{p}$ being non-trivial only in a neighborhood of the point $p$.

Consider, for example, the linear fiber coordinates $v^{i} \in C^{\infty}\left(T_{p} M\right)[[\lambda]]$, viewed as functions on the tangent space. Then $v^{i} \star v^{j}=v^{i} v^{j}+\lambda C_{1}\left(v^{i}, v^{j}\right)+\cdots$ whence $\left[v^{i}, v^{j}\right]_{\star}=\mathrm{i} \lambda \theta_{p}^{i j}+\cdots$. In fact, the star product $\star$ can be chosen in such a way that for the linear coordinates $v^{i}$ the commutator has only the first order terms in $\lambda$. In any case, note that $\theta_{p}^{i j}$ is not a constant but a function on $T_{p} M$ with compact support in $\mathcal{U}_{p}$. On $M$, this yields

$$
\left[x^{i}, x^{j}\right]_{\tilde{\star}_{p}}=\mathrm{i} \lambda \tilde{\theta}_{p}^{i j}+\cdots
$$

for the geodesic normal coordinates $x^{i}$, viewed as local functions on $M$. Here, the coefficient functions $\tilde{\theta}_{p}^{i j}$ are zero outside a neighborhood of $p$ contained in $\mathcal{V}_{p}$. 


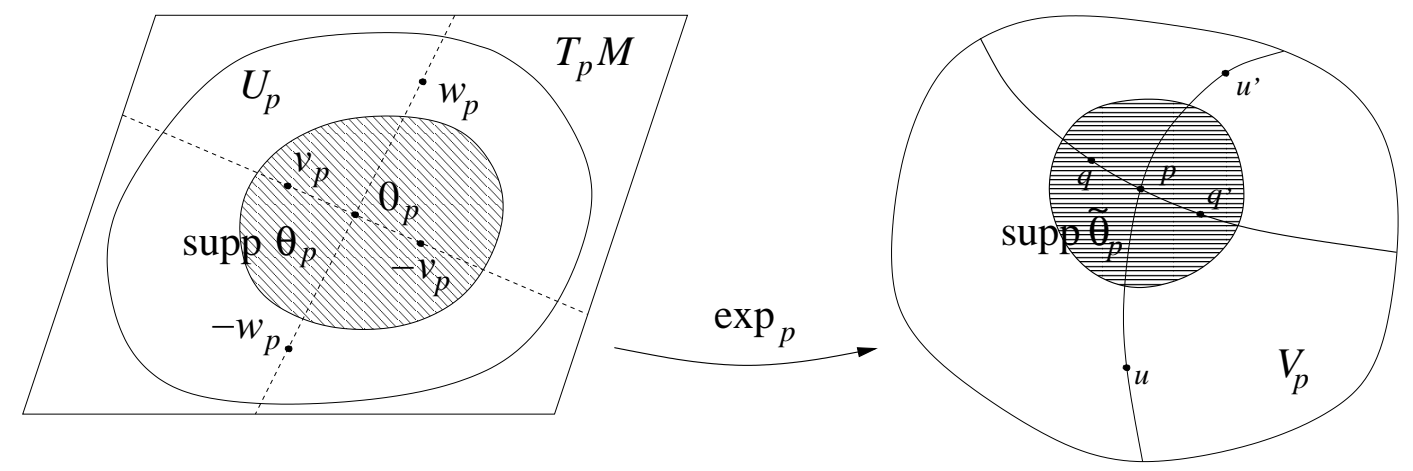

Figure 4: The points $q$ and $q^{\prime}$ are in within the noncommutative bubble around their geodesic midpoint $p$, the points $u$ and $u^{\prime}$ are still outside.

Remark 3.1 Let us emphasize now clearly the interpretation of the star products $\tilde{\star}_{p}$ compared to the usual star products on the space-time manifolds $M$ as mentioned in the introduction. The main difference is that we now have a whole family of star products $\left\{\tilde{\star}_{p}\right\}_{p \in M}$ instead of just one. The interpretation of the algebra $\left(C^{\infty}(M)[[\lambda]], \tilde{\star}_{p}\right)$ comes from the global picture $\left(C^{\infty}(M \times M)[[\lambda]], \tilde{\star}\right)$. We are still discussing observables of two points, i.e. functions $f$ on $M \times M$ and not functions on $M$. However, we may be interested in states, like the $\delta$-functionals $\delta_{q, q^{\prime}}$ on $M \times M$ and their quantum analogs, say for $\left(q, q^{\prime}\right) \in \mathcal{V}$ to make things non-trivial. Then, thanks to verticality, all we have to know to evaluate observables in such a state are their restrictions to the dashed lines in Figure 3 through their geodesic midpoint $p$, see Figure 4 . Then we can equivalently work with $\left(C^{\infty}(M)[[\lambda]], \tilde{\star}_{p}\right)$ and the restriction of the observable $f$ to such a dashed line eventually yields a corresponding function on $M$ via $\iota_{p}^{*}$ and $\left(\exp _{p}\right)_{*}$. Clearly, we have to make precise what notion of states we are going to use.

\subsection{Hermitean vertical star products and their states}

The observables of our theory are functions $f \in C^{\infty}(M \times M)[[\lambda]]$. We will now specify states and the corresponding expectation values for these observables, employing the usual techniques of deformation quantization, which are a straightforward analogue of the well-known approaches in $C^{*}$-algebra or $O^{*}$-algebra theory, see e.g. [32] for a review.

First we make the additional assumption that $\tilde{\star}$ and hence also $\star, \star_{p}$ and $\tilde{\star}_{p}$ are Hermitean, i.e. we require

$$
\overline{f \tilde{\star} g}=\bar{g} \tilde{\star} \bar{f}
$$

for all $f, g \in C^{\infty}(M \times M)[[\lambda]]$, where $\bar{\lambda}=\lambda$ is treated as a real quantity. If we construct $\tilde{\star}$ out of $\tilde{\theta}$ or, equivalently, $\star$ out of $\theta$ using a formality as in Theorem A.13 then the reality $\theta=\bar{\theta}$ of the Poisson structure implies that the corresponding star product is Hermitean. We can therefore safely assume (3.7) for $\tilde{\star}, \star, \star_{p}$ and $\tilde{\star}_{p}$ in the following.

The complex conjugation now being an involution, we define states as positive $\mathbb{C}[[\lambda]]$-linear functionals

$$
\omega: C^{\infty}(M \times M)[[\lambda]] \longrightarrow \mathbb{C}[[\lambda]] \text { with } \omega(\bar{f} \star f) \geq 0,
$$

where the positivity is understood in the sense of formal power series (a real formal power series $a=\sum_{r=r_{0}}^{\infty} \lambda^{r} a_{r} \in \mathbb{R}[[\lambda]]$ is positive, if the lowest non-vanishing coefficient $a_{r_{0}}$ is positive, $a_{r_{0}}>0$ ). 
In addition to (3.8) we require that states be normalized, $\omega(1)=1$. The $\mathbb{C}[[\lambda]]$-linearity implies that $\omega$ is of the form

$$
\omega=\sum_{r=0}^{\infty} \lambda^{r} \omega_{r} \quad \text { with } \mathbb{C} \text {-linear maps } \omega_{r}: C^{\infty}(M \times M) \longrightarrow \mathbb{C} .
$$

In particular, $\omega_{0}$ turns out to be a positive $\mathbb{C}$-linear functional of the commutative ${ }^{*}$-algebra $C^{\infty}(M \times M)$, i.e. $\omega_{0}(\bar{f} f) \geq 0$ for all $f \in C^{\infty}(M \times M)$. It follows that $\omega_{0}$ is the integration with respect to a compactly supported positive Borel measure on $M \times M$.

Conversely, and this is the important point here, one can show that any classical $\omega_{0}$ can be deformed into a functional $\omega$ which is a state with respect to $\tilde{\star}$, see [9]. Note that the 'quantum corrections' $\omega_{r}$ to $\omega_{0}$, which, in general, are necessary to ensure positivity, are by no means unique: there are many quantum states $\omega$ with the same classical limit $\omega_{0}$. Although it is generally very difficult to find the corrections explicitly, one can show that they can always be chosen to be of the form $\omega_{r}=\omega_{0} \circ S_{r}$ with a differential operator $S_{r}$. In such a case, the support of $\omega$ coincides with that of $\omega_{0}$.

Due to the positivity of $\omega$, we may interpret $\omega(f)$ as the expectation value of the observable $f$ in the state $\omega$. Now, $\omega$ still satisfies a Cauchy-Schwarz inequality (in the sense of formal power series), allowing us to write down uncertainty relations. As usual, we define the variance of an observable $f$ in the state $\omega$ by

$$
\operatorname{Var}_{\omega}(f)=\omega(\overline{(f-\omega(f))} \tilde{\star}(f-\omega(f))) \geq 0 .
$$

Then for two Hermitean elements $f=\bar{f}$ and $g=\bar{g}$, i.e. observables in the stricter sense, we find the usual uncertainty relation

$$
4 \operatorname{Var}_{\omega}(f) \operatorname{Var}_{\omega}(g) \geq \omega\left([f, g]_{\tilde{\star}}\right) \overline{\omega\left([f, g]_{\tilde{\star}}\right)},
$$

where the Cauchy-Schwarz inequality for $\omega$ has been used. As usual, these inequalities justify the identification of positive functionals with states.

Remark 3.2 We should note that the notion of positivity we are using is on one hand the only reasonable from an algebraic point of view: it is the unique one which makes $\mathbb{R}[[\lambda]]$ an ordered ring such that $\lambda>0$. On the other hand, there is a more concrete motivation coming from asymptotics: if we think of our formal star product $\star$ as being the asymptotic expansion of some convergent product, say in a $C^{*}$-algebraic approach, then one can also asymptotically expand positive linear functionals which yield precisely the ones we are studying. In this sense, the notion of positivity we are using is the best we can have. See also [32] for a more detailed discussion of states in deformation quantization.

Remark 3.3 It will be important for the physical interpretation to note how the noncommutative structure has entered here: $\omega$ is a deformed classical state $\omega_{0}$ whose quantum corrections depend on $\tilde{\star}$, such that the expectation values of the observable $f$ is changed when we pass from classical to noncommutative space-time. It is the main feature of the deformation approach that the observable $f$ itself remains unchanged: it is still the same function with the same physical interpretation as observable. We only changed the product structure and hence the states.

One effect of noncommutativity is that the variances of observables will in general be strictly larger than the classical ones. In particular, the $\delta$-functionals $\delta_{\left(q, q^{\prime}\right)}$ for $\left(q, q^{\prime}\right) \in M \times M$ are no longer positive with respect to $\tilde{\star}$, but require quantum corrections, and we will always find observables 
such that the variances in these deformed $\delta$-functionals are strictly positive (while the classical ones are of course 0). We shall come back to explicit examples in Section 4

Let us now discuss why our model meets the physical requirements which we have argued for. We consider now a classical state $\omega_{0}$, i.e. a positive Borel measure on $M \times M$ whose (compact) support supp $\omega_{0}$ is far away from the diagonal $\Delta_{M}$, and in particular, $\operatorname{supp} \omega_{0} \cap \operatorname{supp} \tilde{\theta}=\emptyset$. It immediately follows that $\omega_{0}$ is a state with respect to $\tilde{\star}$, since $\tilde{\star}$ is non-trivial only in $\operatorname{supp} \tilde{\theta}$. Indeed, we have

$$
\omega_{0}(f \tilde{\star} g)=\omega_{0}(f g)
$$

for all $f, g \in C^{\infty}(M \times M)[[\lambda]]$ in this case. Therefore, all variances and covariances of $f$ and $g$ with respect to $\omega_{0}$ are the classical ones. In particular, only the classical variances appear in (3.11) and the right hand side is zero, although $[f, g]_{\tilde{\star}}$ may be different from zero. This shows that if we evaluate observables $f \in C^{\infty}(M \times M)[[\lambda]]$ far away from the diagonal, no noncommutative behavior can be seen. The noncommutativity only appears close to the diagonal as is expected from the support conditions on $\tilde{\theta}$ and $\tilde{\star}$. This is precisely the behavior we wanted. At large distances our locally noncommutative space-time behaves entirely classically.

\subsection{Distance measurements and the causal structure}

Let us now reconsider the interpretation of our noncommutative structure from the point of view of distance measurements. Since the concept of 'distance' is of course misleading in a pseudoRiemannian context, we shall not measure a distance function, but measure the metric directly. It turns out that this can be done most natural in our framework.

As a motivation one may think of a Riemannian situation where the metric distance $d\left(q, q^{\prime}\right)$ between two points $q$ and $q^{\prime}$ is defined as the infimum over the lengths of all paths joining the two points. In general, this is a highly non-trivial quantity. However, if the points are close enough then one finds a unique shortest geodesic joining them, whose length realizes $d\left(q, q^{\prime}\right)$. In fact, if $\left(q, q^{\prime}\right) \in \mathcal{V}$ then this is the case and the geodesic is precisely the one starting from the geodesic midpoint $p$ in opposite directions where $-v_{p}=\exp _{p}^{-1}(q)$ and $v_{p}=\exp _{p}^{-1}\left(q^{\prime}\right)$. In this case, the distance is given by $d\left(q, q^{\prime}\right)=2 \sqrt{g_{p}\left(v_{p}, v_{p}\right)}$. In particular, the square of the distance function is the smooth function $d^{2}\left(q, q^{\prime}\right)=4 g_{p}\left(v_{p}, v_{p}\right)$. In general, the distance function is only smooth close to the diagonal.

In the general situation we shall therefore use the function $d^{2} \in C^{\infty}(T M)$ defined by

$$
d^{2}\left(v_{p}\right)=g_{p}\left(v_{p}, v_{p}\right)
$$

as a good replacement for the geodesic distance function. It is a quadratic function on $T M$ which is everywhere smooth and in the neighborhood $\mathcal{U}$ it is indeed the square of the distance function in the Riemannian case. Since we are only interested in the behavior close to the diagonal $\Delta_{M}$ as the noncommutativity is only present here, this will be a perfect observable to measure the metric.

The quantum effects will now come into the game in the expectation values of this observable $d^{2}$ if we evaluate it in some state. In particular, we are interested in those states which are as close as possible to the $\delta$-functionals at some point $v_{p}$. Thanks to our verticality condition we can consider even the restricted situation, i.e. the observable $\iota_{p}^{*} d^{2} \in C^{\infty}\left(T_{p} M\right)[[\lambda]]$. Then we need a deformation of the $\delta$-functional

$$
\delta_{v_{p}}^{\left(\star_{p}\right)}=\delta_{v_{p}} \circ S_{v_{p}}, \quad \text { where } \quad S_{v_{p}}=\mathrm{id}+\sum_{r=1}^{\infty} \lambda^{r} S_{v_{p}}^{(r)},
$$


into a positive functional for $\star_{p}$. Then the quantum distance square between $q$ and $q^{\prime}$ is now (up to the factor 4) the evaluation

$$
\delta_{v_{p}}^{\left(\star_{p}\right)}\left(d^{2}\right)=d^{2}\left(v_{p}\right)+\lambda\left(S_{v_{p}}^{(1)} d^{2}\right)\left(v_{p}\right)+\cdots,
$$

which is clearly a deformation of the classical distance square. Moreover, in general we obtain a non-trivial variance of this measurement according to (4.13) since on one hand $d^{2} \star_{p} d^{2}$ is not just the pointwise product and on the other hand the correction terms $S_{v_{p}}^{(r)}$ contribute as well. This way we arrive at the observation that the geometry indeed becomes fuzzy. Note however, that the choice for a deformation of $\delta_{v_{p}}$ is not unique at all.

Let us also remark already at this point that in the Lorentz situation the sign of the classical evaluation $d^{2}\left(v_{p}\right)$ determines whether $v_{p}$ is a space-like, light-like or time-like vector. In our case, this characterization needs no longer to be valid, in particular, the light-like vectors with $d^{2}\left(v_{p}\right)=0$ might get correction terms from the deformed $\delta$-functional which makes them space-like or time-like. Note however, that this again depends of course on our choice of the deformation $\delta_{v_{p}}^{\left(\star_{p}\right)}$ : this simply reflects again that there are no 'classical' points any more in a truly noncommutative space-time.

\section{The Noncommutative Minkowski Space}

In order to analyze our construction's properties more explicitly, we now discuss the case where $M$ is a vector space of dimension $n$ in more detail. We chose $\nabla$ to be the canonical flat connection. Then the exponential map at each point is a global diffeomorphism, and so is $\Phi$. In fact, the exponential map implements a diffeomorphism of $T_{p} M$ to $M$ given by the translation,

$$
\exp _{p}(v)=p+v \in M \quad \forall v \in T_{p} M
$$

and the local formulae of the previous section are now globally defined. In particular, for any pair of points $\left(q, q^{\prime}\right) \in M \times M$, the midpoint $p=\left(q+q^{\prime}\right) / 2$ and the relative coordinates $v=\left(-q+q^{\prime}\right) / 2$ are now globally defined. For this reason, the dashed lines from Figure 3 which denote the geodesic relative coordinates and in the general case are defined only in some open neighborhood $\mathcal{V} \subset M \times M$ of the diagonal $\Delta_{M}$, now become straight lines extending to infinity, see Figure 5 . Note also, that up to now we have only used the connection $\nabla$ but no metric.

Let $f, g \in C^{\infty}(T M)[[\lambda]]$, then

$$
f \star g=\sum \lambda^{r} C_{r}(f, g),
$$

where

$$
C_{r}(f, g)=\sum_{I, J} C_{r}^{I J} \frac{\partial^{|I|} f}{\partial v^{I}} \frac{\partial^{|J|} g}{\partial v^{J}},
$$

with multi indices $I, J \subset\{1, \ldots, n\},|I|,|J| \leq r$. Note that $C_{r}$ differentiates only in direction of the tangent spaces, i.e. in $v$-directions. Thanks to the simple form of the exponential map (4.1) we find from (2.6) for all $f, g \in C^{\infty}(M \times M)[[\lambda]]$

$$
f \tilde{\star} g=\sum \lambda^{r} \sum_{I, J} \tilde{C}_{r}^{I J} \prod_{i \in I}\left(-\frac{\partial}{\partial q^{i}}+\frac{\partial}{\partial q^{\prime i}}\right) f \prod_{j \in J}\left(-\frac{\partial}{\partial q^{j}}+\frac{\partial}{\partial q^{\prime j}}\right) g
$$

where $\tilde{C}_{r}^{I J}=C_{r}^{I J} \circ \Phi^{-1}$. Here, we have used that $\partial_{v}\left(\Phi^{*} f\right)(p, v)=\left(\left(-\partial_{1}+\partial_{2}\right) f\right)(\Phi(p, v))$ with $\partial_{i}$ denoting the derivative with respect to the $i^{\text {th }}$ argument. 


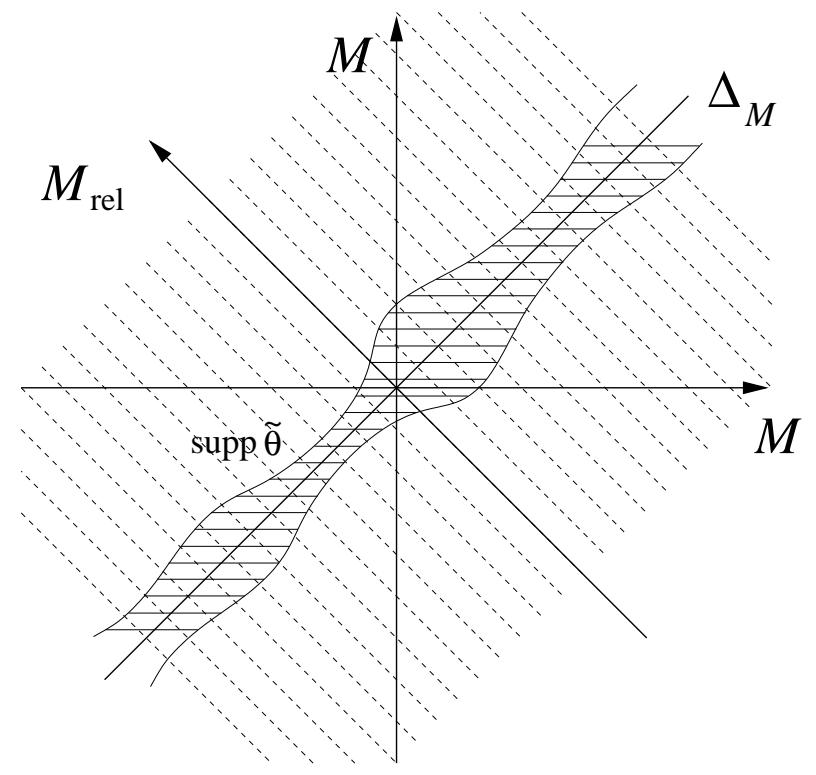

Figure 5: In the case of Minkowski space the map $\Phi$ is a global diffeomorphism and introduces the global center of mass and relative coordinates on $M \times M$.

\subsection{Global constant Poisson structure}

As $\Phi$ is a global diffeomorphism on flat space, there is in principle no need to restrict the range of noncommutativity, i.e. to have $\tilde{\theta}$ compactly supported. We will however, of course choose to still impose such restrictions in order to implement localized noncommutativity, see Figure 5. But for the time being, in order to compare our approach to more commonly analyzed settings, we now restrict ourselves to the special case of a constant vertical Poisson structure

$$
\theta=\frac{1}{2} \theta^{i j} \frac{\partial}{\partial v^{i}} \wedge \frac{\partial}{\partial v^{j}} \quad \text { with } \quad \theta^{i j}=-\theta^{j i} \in \mathbb{R} .
$$

Then, the star product on $T M$ can be chosen to be the usual Weyl-Moyal product

$$
f \star g=\sum_{r=0}^{\infty} \frac{1}{r !}\left(\frac{\mathrm{i} \lambda}{2}\right)^{r} \theta^{i_{1} j_{1}} \cdots \theta^{i_{r} j_{r}} \frac{\partial^{r} f}{\partial v^{i_{1}} \cdots v^{i_{r}}} \frac{\partial^{r} g}{\partial v^{j_{1}} \cdots v^{j_{r}}} .
$$

It is obviously invariant under reflections in the sense of (2.7). With such a constant Poisson structure, any pair of points is within the range of noncommutativity of their midpoint, since $\operatorname{supp} \tilde{\theta}_{p}=T_{p} M$.

Clearly, the dependence on $p$ is only in the functions $f$ and $g$ and we recognize that (4.6) restricts to the Weyl-Moyal star product on $T_{p} M$ with respect to $\theta$, i.e.

$$
f \star_{p} g=\mu \circ \exp \left(-\frac{\mathrm{i} \lambda}{2} \theta^{i j} \partial_{v^{i}} \otimes \partial_{v^{j}}\right)(f \otimes g)
$$

for $f, g \in C^{\infty}\left(T_{p} M\right)[[\lambda]]$ where $\mu(f \otimes g)=f g$ is the usual pointwise product. Although in fact, the star product is independent of $p$, we keep the notation $\star_{p}$ in order to remember that we are considering some fixed $T_{p} M$. Likewise, we find for $f, g \in C^{\infty}(M \times M)[[\lambda]]$

$$
f \tilde{\star} g=\mu \circ \exp \left(\frac{\mathrm{i} \lambda}{2} \theta^{i j}\left(-\frac{\partial}{\partial q^{i}}+\frac{\partial}{\partial{q^{\prime}}^{i}}\right) \otimes\left(-\frac{\partial}{\partial q^{j}}+\frac{\partial}{\partial q^{\prime j}}\right)\right)(f \otimes g) .
$$


Obviously, the differentiation is in the direction of the line through $q$ and $q^{\prime}$ (i.e. perpendicular to the diagonal).

As in Section 3.2 we now ask ourselves how close two points $\left(q, q^{\prime}\right) \in M \times M$ may be to one another. In order to do so, we consider the situation on $T_{p} M$, and modify the $\delta$-distribution such that it is a positive functional with respect to the star product $\star_{p}$. As discussed in Section 3.1 this deformation is not unique, but as a natural candidate we use the formal version of the coherent states of quantum mechanics, see the discussion in $[8,9]$. For simplicity we assume that $\theta$ is nondegenerate, i.e. a symplectic Poisson tensor. Hence in particular, $M$ has to be even dimensional. Then we consider

$$
\delta^{\left(\star_{p}\right)}=\delta \circ \mathrm{e}^{\frac{1}{4} \lambda \Delta_{g}}
$$

and likewise for the translates of the $\delta$-distribution $\delta_{w}, w \in M$. Here, $g^{-1}$ is a positive compatible scalar product with $\theta$, i.e. there exists a linear complex structure $J \in \operatorname{End}\left(T_{p} M\right), J^{2}=-$ id with $g^{-1}(v, w)=\omega_{\theta}(v, J w)$ for all $v, w \in T_{p} M$, where $\omega_{\theta}$ is the associated symplectic form to $\theta$. Finally, $\Delta_{g}$ denotes the usual Laplacian with respect to $g^{-1}$. In the following we shall mainly consider the standard symplectic form $\theta$ and chose for $g^{-1}$ the identity matrix with respect to some given choice of Darboux coordinates on $T_{p} M$.

Now, for any quadratic form $A \in M(n, \mathbb{R}), f_{A}(v)=v^{t} A v$ and for a Laplacian $\Delta_{g}$ with respect to some symmetric form $g^{-1}$, we find

$$
\mathrm{e}^{\frac{1}{4} \lambda \Delta_{g}} f_{A}=f_{A}+\frac{\lambda}{2} \operatorname{tr}(g A)
$$

and, after a short calculation,

$$
\mathrm{e}^{\frac{1}{4} \lambda \Delta_{g}}\left(f_{A} \star f_{A}\right)=f_{A}^{2}+\lambda f_{A} \operatorname{tr} g A+2 \lambda f_{A g A}+\frac{\lambda^{2}}{4}\left(2 \operatorname{tr}\left(A_{\theta} A_{\theta}\right)+(\operatorname{tr} g A)^{2}+2 \operatorname{tr} g A g A\right),
$$

where $\left(A_{\theta}\right)_{j}^{r}=\theta^{r s} A_{s j}$ and where we have used $\Delta_{g}\left(f_{A} f_{A}\right)=4 f_{A}(\operatorname{tr} g A)+8 f_{A g A}$ and $\Delta_{g}^{2}\left(f_{A} f_{A}\right)=$ $8(\operatorname{tr} g A)^{2}+16 \operatorname{tr} g A g A$.

Following our general discussion in Section 3.2 let us now investigate the Lorentz square in 4 dimensions, i.e. consider $f_{\eta}$ where $\eta=\operatorname{diag}(+,-,-,-)$ and $n=4$. By (4.10), a distance measurement as above yields for the squared distance,

$$
\delta_{v_{p}}^{\left(\star_{p}\right)}\left(f_{\eta}\right)=f_{\eta}\left(v_{p}\right)+\frac{\lambda}{2} \operatorname{tr}(\eta g)=\eta\left(v_{p}, v_{p}\right)-\lambda,
$$

whence all Lorentz squares acquire a negative offset independent of $v_{p}$ in this particular deformation of the classical $\delta$-functional. The resulting deformed light cone on $T_{p} M$, defined by $\delta_{v}^{\left(\star_{p}\right)}\left(f_{\eta}\right)=0$, then takes the form of two hyperbolae, $v_{0}= \pm \sqrt{\lambda+\vec{v}^{2}}$ (i.e. a "mass shell" of mass $\lambda$ ), approaching the ordinary light-cone for distances $\|v\| \gg \lambda$, see Figure 6. The time-like vectors are characterized by $v_{0}^{2}>\lambda+\vec{v}^{2}$, and the space-like vectors are those with $v_{0}^{2}<\lambda+\vec{v}^{2}$. To interpret this picture we recall that the point $p$ does not have meaning in itself, but only as the geodesic midpoint of two other points $\exp _{p}\left( \pm v_{p}\right)=p \pm v_{p}$, such that the points $v_{p}$ and $-v_{p}$ are connected by a time-/spaceor light-like line (in the deformed sense). The gap between future and past time-like lines around $0_{p}$ is of the order $\lambda$ and is to be interpreted as follows: if the two points in $M, p \pm v_{p}$, approach each other, the causal structure is lost. This is however, by construction in accordance with our minimal resolvable distance.

Note that the offset would have opposite sign, had we used $\eta=\operatorname{diag}(-,+,+,+)$, so also in this case, the light-cone is deformed in the same manner as above. For the variance we find

$$
\operatorname{Var}_{\delta_{v_{p}}^{(\star p)}}\left(f_{\eta}\right)=f_{\eta^{2}}\left(v_{p}\right)+2 \lambda^{2}
$$

by (4.11) and the fact that $f_{\eta} \star_{p} f_{\eta}=f_{\eta} f_{\eta}$ since $\sum_{r, j}\left(\eta_{\theta}\right)_{j}^{r}\left(\eta_{\theta}\right)_{r}^{j}=-\sum\left(\theta^{r j}\right)^{2} \eta_{j j} \eta_{r r}=0$. 


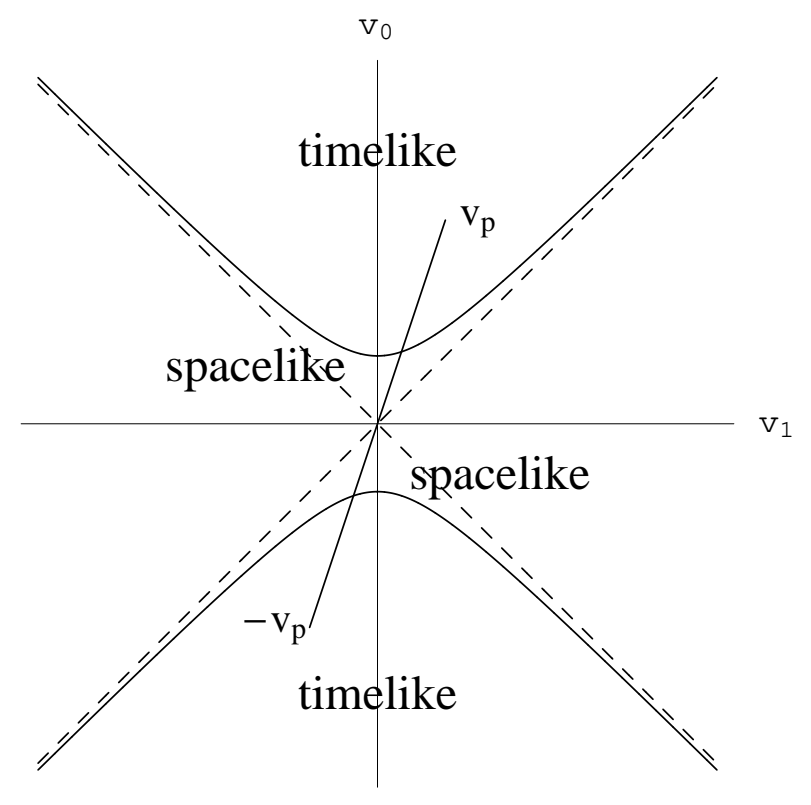

Figure 6: The ordinary and the deformed light-cone in the case of constant $\theta$. Two spatial dimensions of $v \in T_{p} M$ are suppressed.

Remark 4.1 We also would like to note that the measurements of the distance square are not an artifact of our approach but an intrinsic feature of the noncommutative Minkowski space-time with constant $\theta$.

\subsection{Non-constant Poisson structures}

In a slightly more general scenario, we might want to employ a Poisson structure which is constant along each fiber $T_{p} M$, but varies depending on the absolute position of the center of mass, $p$. In this case, the formulae (4.6) and (4.8) from the previous discussion remain valid; the only difference being that $\theta^{i j}$ now explicitly depends on $p$. This however, seems to be a rather unnatural scenario, since translation invariance is unnecessarily broken. In any case, we set out to construct a noncommutative structure that vanishes in the limit of large distances. In particular, we require that an appropriate deformation $\delta_{q, q^{\prime}}^{(\widetilde{\star})}$ of the $\delta$-Distribution on $C^{\infty}(M \times M)$, fulfills (3.12) for $\left(q, q^{\prime}\right)$ far away from the diagonal $\Delta_{M}$, i. e. that for $f, g \in C^{\infty}(M \times M)[[\lambda]], \delta_{q, q^{\prime}}^{(\tilde{\star})}(f \tilde{\star} g)=\delta_{q, q^{\prime}}(f g)$ (or equivalently, on $T_{p} M$, that for $f, g \in C^{\infty}\left(T_{p} M\right)[[\lambda]], \delta_{v_{p}}^{\left(\star_{p}\right)}\left(f \star_{p} g\right)=\delta_{v_{p}}^{\left(\star_{p}\right)}(f g)$ for large $\left.v_{p}\right)$. Obviously, this is not true for a nontrivial Poisson structure that is constant along the fiber $T_{p} M$ whether it depends on $p$ in a non-trivial way or not.

Let us therefore now turn to a scenario which actually exhibits the features our more general approach allows for and choose $\operatorname{supp} \theta_{p}$ to be compact. For concreteness' sake we may think of the special Poisson structure for which $\theta_{p}$ is constant on a ball $\overline{B_{r}\left(0_{p}\right)}$ around $0_{p} \in T_{p} M$ and then decreases quickly to 0 , such that $\operatorname{supp} \theta_{p} \subset \overline{B_{r+\epsilon}\left(0_{p}\right)}$ for some $\epsilon$. To implement flip symmetry (2.5) we moreover impose that $\theta_{p}^{i j}\left(v_{p}\right)=\theta_{p}^{i j}\left(-v_{p}\right)$ for all $v_{p} \in T_{p} M$ (on $\overline{B_{r}\left(0_{p}\right)}$ this is of course trivially fulfilled). Such Poisson structures exist, see Example A.12 and meet all our requirements. Clearly, we can use the same such $\theta_{p}$ for all $p$ whence we easily can implement translation invariance. In particular, the support $\operatorname{supp} \theta \cap T_{p} M$ is of the same size for all $p \in M$. 
In such a scenario we again consider a distance measurement. Although the deformation $\delta_{v_{p}} \circ$ $\mathrm{e}^{\frac{1}{4} \lambda \Delta_{g}}$ we previously employed, may no longer be a positive functional for $\left(C^{\infty}\left(T_{p} M\right)[[\lambda]], \star_{p}\right)$ for all classical points $v_{p} \in T_{p} M$, it will be positive for $v_{p}$ contained in $\overline{B_{r}\left(0_{p}\right)}$. Here, $\theta_{p}$ is constant, and the star product coincides with the Weyl-Moyal star product as in (4.6). If we are interested only in smallest distances, this set of states is sufficient and evaluating the function $f_{\eta}$ in $\delta_{v_{p}}^{\star_{p}}$ with $v_{p} \in B_{r}\left(0_{p}\right)$, we gain the same results as in the case of globally constant $\theta_{p}$ above. Of course, if we consider states corresponding to $\delta$-functionals $\delta_{v_{p}}$ for larger $v_{p}$, we will have to consider some other deformation. Moreover, in the area where $\theta_{p}$ drops to zero as a function of $v_{p}$, the additional derivatives of $\theta_{p}$ will contribute significantly to the distance measurement, see also Figure 7 . Thus,

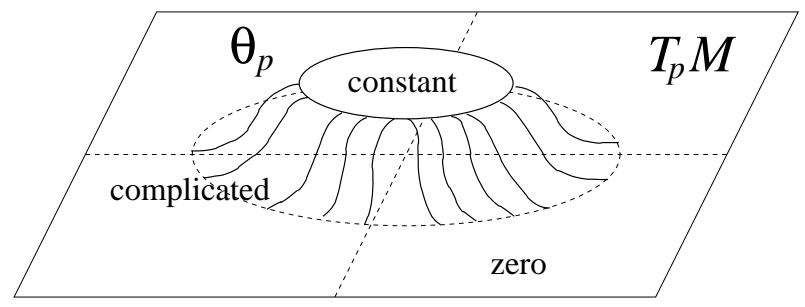

Figure 7: Schematic view of a Poisson structure $\theta_{p}$ being constant around $0_{p}$ with compact support

it will be of major importance to understand the state space of formal star products better. Hence, a priori the passage from very small to large distances is not yet very well controlled in such models. We hope to come back to this question at a later stage.

\subsection{Symmetries}

It is natural now to consider the behavior of our construction with respect to Poincaré transformations. In the discussion above we have already seen that translation invariance can easily be accounted for by choosing $\theta_{p}$ and $\star_{p}$ to be independent of the geodesic midpoint $p$, i.e. to choose the same structure on all $T_{p} M$. It is furthermore quite simple to implement invariance of the star product under orthogonal transformations with respect to some positive definite scalar product, simply by asking that $R^{*} \theta_{p}=\theta_{p}$ for all $R \in O(4)$. Note that reflections at $0_{p}$ are already taken care of by implementing the flip symmetry.

Lorentz symmetry on the other hand, cannot be implemented in such a simple manner. The reason is that on one hand the diagonal action of the Lorentz group on $M \times M$ induces the usual action on the relative coordinates in $T_{p} M$. On the other hand, it is well-known that there is no Lorentz invariant antisymmetric bivector on $T_{p} M$ beside $\theta_{p}=0$. Thus we necessarily break Lorentz invariance already on the semi-classical level of $\theta_{p}$.

For a constant and also translation invariant $\theta_{p}$ we might however mimic the approach taken in [15] and consider along with some fixed $\theta_{p}$ the whole orbit under Lorentz transformations. Thus with this covariant transformation law for $\theta_{p}$ we would obtain an action of the Lorentz group on the whole algebra but of course we have introduced this additional orbit which affects the classical limit in a non-trivial way [15].

\section{$5 \quad$ Further Questions and Outlook}

Let us conclude with some open questions and further remarks. 


\subsection{Dynamics and Field Theories}

Up to now we have only set up a kinematic framework for a locally noncommutative space-time. This is of course not in the least enough to have a reasonable model for space-time at small distances: We certainly have to include some sort of dynamics into our description. Here one should try to proceed in the usual stages.

A first approach would be to investigate the behaviour of point-like classical or quantum mechanical particles moving in such a locally noncommutative space-time. Especially for non-relativistic considerations, it seems reasonable to consider as a first step only a locally noncommutative space and treat time as an ordinary real parameter.

In a second step, one can consider classical field theories on our locally noncommutative spacetime. A good starting point is provided by deformed vector bundles in the sense of $[7,30,31]$ concerning the matter part. It is however, not yet clear how to define interaction terms, though one might hope to do so using suitably deformed Hermitian fiber metrics. For gauge fields one can then rely on $[20,21]$, formulated in a suitable geometric fashion.

In a third step, one wants to construct quantum field theories corresponding to the classical field theories on a locally noncommutative space-time. We shall discuss this in more detail in the next section.

Note however, that for a consistent dynamical treatment we have to go at least one step further: the Poisson structure $\theta$ itself (and hence the corresponding star product) should be considered as a dynamical quantity instead of a fixed background field. This is of course desirable in any model of noncommutative space-time and therefore poses a general open problem in such approaches to Planck scale physics. Here, the principle difficulty is to impose field equations on $\theta$ which are compatible with the Jacobi identity $\llbracket \theta, \theta \rrbracket=0$ and still allow some interesting coupling to other fields.

\subsection{Quantum Field Theories}

We continue with some heuristic remarks on possible quantum field theories on a locally noncommutative space-time.

The main goal of our construction is the avoidance of a violation of locality at large distances in field theory. To get a first glimpse on how powerful our ideas might turn out to be, consider the flat Minkowski space $M$ with a star product on $M \times M$ meeting our requirements of compact support in relative coordinate directions. Let us assume that the free field $\phi(x)$ is the ordinary one, i.e. $\phi(x)=\int \hat{\phi}(k) \mathrm{e}^{i k x} \mathrm{~d} k$ with the operator valued distribution $\hat{\phi}(k)$ acting on Fock space as annihilation and creation operators, respectively.

Now consider two fields at different points $x$ and $y$ in space time (to be precise, we have to evaluate $\phi$ in suitable test functions supported around $x$ and $y$ ). In order to fit them into our framework, we consider the operator-valued distributions

$$
f(x, y)=\phi(x) \quad \text { and } \quad g(x, y)=\phi(y)
$$

Using the globally defined exponential map, we now define for $p=\frac{x+y}{2}$ and $v=\frac{x-y}{2}$

$$
F(p, v)=\phi(p+v) \quad \text { and } \quad G(p, v)=\phi(p-v)
$$

and consider their star product (ignoring all problems that arise in taking such products of distributions). We then find that for $v \notin \operatorname{supp} \theta_{p}$, the star product becomes the ordinary one and in particular, the commutator of fields is unchanged,

$$
[F, G]_{\star_{p}}(p, v)=[\phi(p+v), \phi(p-v)]_{\star_{p}}=[\phi(p+v), \phi(p-v)] \quad \text { for } \quad v \notin \operatorname{supp} \theta_{p}
$$


Clearly, for $v$ large enough we thus recover micro-locality (in particular, vanishing of the commutator for space-like separated points). The classic no-go theorems on nonlocal fields [5,26] are circumvented as the product of the fields itself is changed.

It remains an open and difficult problem to define a sensible interaction term. Possibly, one should employ the deformed $\delta$-distributions (much in the spirit of [2]) or try to generalize the approach to more than two points.

Our hope is however, that once this has been achieved, the field theory's properties regarding renormalization should be considerably improved compared to both the ordinary one as well as the one based on models with constant $\theta$ (as the infrared regime is clearly separated from the ultraviolet one). In the long run, it would have to be investigated whether a quickly decreasing (instead of a compactly supported) noncommutative structure would suffice for the purposes of renormalization such that problems with the Lorentz structure could be avoided - though the construction possibly only admits this for flat space (where the exponential map defines a global diffeomorphism).

\subsection{Further extensions of the model}

Let us finally mention some possible extensions of the locally noncommutative space-times as presented above. Once having realized that not $M$ but $M \times M$ is relevant when discussing small distance behavior one can of course go one step further: In principle one can also discuss noncommutativity which only becomes present when three points come close together. This would give a noncommutativity on $M \times M \times M$ located again close to the diagonal. Analogously, one can consider Poisson structures $\theta^{(k)}$ on $M^{k}=M \times \cdots \times M$ for arbitrary $k \in \mathbb{N}$ with support close around the total diagonal. It would be clearly a very interesting investigation how one can combine all these $\theta^{(k)}$ and formulate compatibilities between them for different $k$. In particular, it would be interesting to find a reasonable replacement for the verticality requirement.

The last extension we want to mention is the passage from formal star products to convergent deformations. This is known to be a serious problem in deformation quantization and not much can be said on a general level. However, for certain Poisson structures there exist convergent star products quantizing them, usually by means of suitable integral formulas, see e.g. [4,23, 27]. Eventually, the result will be a $C^{*}$-algebraic approach like in [15] which will be necessary for all questions concerning quantum field theories in the locally noncommutative space-time. For vertical Poisson structures $\theta$ arising from actions of some $\mathbb{R}^{d}$ one can rely on Rieffel's general construction [27] to obtain a $C^{*}$-algebraic deformation. This will be investigated in a future project [19].

\section{A Vertical formality and vertical star products}

In this appendix we collect some results on star products on vector bundles which seem to be new but follow essentially in a straightforward manner from Kontsevich's formality theorem for $\mathbb{R}^{d}$. Thus we only indicate the proofs and outline the ideas.

\section{A.1 Vertical multivector fields on a vector bundle}

Let us first recall some standard results in order to fix our notation. We consider a real vector bundle $\pi: E \longrightarrow M$ with with fiber dimension $d$.

Recall that a tangent vector $X \in T_{v} E$ at $v \in E$ is called vertical if $T_{v} \pi X=0$. The subbundle of vertical tangent vectors is denoted by $\operatorname{Ver}(E) \subseteq T E$. Moreover, we obtain vertical contravariant tensors $\bigotimes^{k} \operatorname{Ver}(E) \subseteq \bigotimes^{k} T E$ as well, in particular the vertical symmetric and antisymmetric 
contravariant tensor bundles $\mathrm{S}^{k} \operatorname{Ver}(E) \subseteq \mathrm{S}^{k} T E$ and $\Lambda^{k} \operatorname{Ver}(E) \subseteq \Lambda^{k} T E$, respectively. The corresponding sections are the vertical contravariant tensor fields $\Gamma^{\infty}\left(\bigotimes^{k} \operatorname{Ver}(E)\right) \subseteq \Gamma^{\infty}\left(\bigotimes^{k} T E\right)$ where we are most interested in the vertical multivector fields $\mathfrak{X}_{\text {ver }}^{\bullet}(E)=\Gamma^{\infty}\left(\Lambda^{\bullet} \operatorname{Ver}(E)\right)$.

In the sequel we make use of local expressions. Thus fix a locally defined basis of sections $e_{1}, \ldots, e_{d} \in \Gamma^{\infty}\left(\left.E\right|_{U}\right)$ where $U \subseteq M$ is a suitable open subset and $\left.E\right|_{U}=\pi^{-1}(U)$. The corresponding dual basis is denoted by $e^{1}, \ldots, e^{d} \in \Gamma^{\infty}\left(\left.E^{*}\right|_{U}\right)$. The choice of such a basis induces linear coordinates $s^{1}, \ldots, s^{d} \in C^{\infty}\left(\left.E\right|_{U}\right)$ along the fibers by setting $s^{\alpha}(v)=\left\langle e^{\alpha}(\pi(v)), v\right\rangle$ as usual, locally trivializing $\left.E\right|_{U} \cong U \times \mathbb{R}^{d}$. If $\left(x^{1}, \ldots, x^{n}\right)$ are local coordinates on $U \subseteq M$ then $\left(x^{1} \circ \pi, \ldots, x^{n} \circ \pi, s^{1}, \ldots, s^{d}\right)$ are local coordinates of $E$ defined on $\left.E\right|_{U}$. The local tangent vector fields $\frac{\partial}{\partial s^{\alpha}}$ are vertical and provide a basis of sections for $\left.\operatorname{Ver}(E)\right|_{U}$.

We can lift sections $s \in \Gamma^{\infty}(E)$ to vertical vector fields $s^{v} \in \mathfrak{X}_{\text {ver }}^{1}(E)$ by setting

$$
s^{\vee}(v)=\left.\frac{\mathrm{d}}{\mathrm{d} t}\right|_{t=0}(v+t s(\pi(v)))
$$

for $v \in E$, whence clearly $\frac{\partial}{\partial s^{\alpha}}=e_{\alpha}^{v}$. Hence the tangent vector fields $\frac{\partial}{\partial s^{\alpha}}$ do not depend on the choice of the local coordinates $\left(x^{1}, \ldots, x^{d}\right)$ but only on the frame $e_{1}, \ldots, e_{d}$.

We can extend the vertical lift to arbitrary tensor fields $X \in \Gamma^{\infty}\left(\otimes^{k} E\right)$ in the usual way, compatible with the tensor product, where a 0-tensor field $u \in \Gamma^{\infty}\left(\bigotimes^{0} E\right)=C^{\infty}(M)$ is lifted via $u^{\vee}=\pi^{*} u$. Locally, any vertical tensor field $X \in \Gamma^{\infty}\left(\bigotimes^{k} \operatorname{Ver}(E)\right)$ can be written as

$$
\left.X\right|_{\pi^{-1}(U)}=X^{\alpha_{1} \cdots \alpha_{k}} e_{\alpha_{1}}^{\mathrm{v}} \otimes \cdots \otimes e_{\alpha_{k}}^{\mathrm{v}}
$$

with $X^{\alpha_{1} \cdots \alpha_{k}} \in C^{\infty}\left(\pi^{-1}(U)\right)$. Then $X$ is a vertical lift iff the functions $X^{\alpha_{1} \cdots \alpha_{k}}$ are pull-backs of functions in $C^{\infty}(U)$. Denote by $\xi \in \Gamma^{\infty}(\operatorname{Ver}(E))$ the Euler vector field, defined via its flow $(t, v) \mapsto$ $\mathrm{e}^{t} v$. Locally, $\left.\xi\right|_{\pi^{-1}(U)}=s^{\alpha} \frac{\partial}{\partial s^{\alpha}}$ and hence $X \in \Gamma^{\infty}\left(\bigotimes^{k} \operatorname{Ver}(E)\right)$ is a vertical lift iff $\mathscr{L}_{\xi} X=-k X$.

More generally, we say that $X \in \Gamma^{\infty}\left(\bigotimes^{k} \operatorname{Ver}(E)\right)$ is polynomial along the fibers of degree $\ell$ if $\mathscr{L}_{\xi} X=(\ell-k) X$. Clearly, this is equivalent to the local statement that all coefficient functions $X^{\alpha_{1} \cdots \alpha_{k}}$ are polynomials in the fiber variables $s^{1}, \ldots, s^{d}$ of degree $\ell$. The vertical tensor fields $X \in \Gamma^{\infty}\left(\bigotimes^{k} \operatorname{Ver}(E)\right)$ polynomial along the fibers of degree $\ell$ are in canonical bijection to tensor fields $\widetilde{X} \in \Gamma^{\infty}\left(\mathrm{S}^{\ell} E^{*} \otimes \bigotimes^{k} E\right)$ via the relation

$$
X(v)=\left(\left.\widetilde{X}\right|_{\pi(v)}(v, \ldots, v)\right)^{v}
$$

where we first insert the point $v \in E_{\pi(v)}$ in the $\mathrm{S}^{\ell} E^{*}$-part $\ell$-times and then lift the $\bigotimes^{k} E$-part vertically. In particular, vertical lifts are those vertical tensor fields which are constant along the fibers.

A vertical tensor field $X \in \Gamma^{\infty}\left(\bigotimes^{k} \operatorname{Ver}(E)\right)$ can be restricted to a fiber $E_{p} \subseteq E$ for $p \in M$ and yields a tensor field in $\Gamma^{\infty}\left(\bigotimes^{k} T E_{p}\right)$. This follows from the fact that canonically $\operatorname{ker} T_{v} \pi \cong T_{v}\left(E_{p}\right)$ for $v \in E_{p}$. Let $\iota_{p}: E_{p} \hookrightarrow E$ denote the inclusion map then we denote the restriction by $\iota_{p}^{*} X \in$ $\Gamma^{\infty}\left(\bigotimes^{k} T E_{p}\right)$. In particular, if $X \in \Gamma^{\infty}\left(\bigotimes^{k} \operatorname{Ver}(E)\right)$ is polynomial along the fibers of degree $\ell$ then $\iota_{p}^{*} X$ is a tensor field on the vector space $E_{p}$ which is polynomial of degree $\ell$ in the usual sense.

Finally, we focus on vertical multivector fields. The following is folklore and consists in a straightforward verification:

Proposition A.1 Let $\pi: E \longrightarrow M$ be a vector bundle.

i.) The vertical multivector fields $\mathfrak{X}_{\mathrm{ver}}^{\bullet}(E)$ are a Gerstenhaber subalgebra of $\mathfrak{X}^{\bullet}(E)$. 
ii.) The restriction map $\iota_{p}^{*}: \mathfrak{X}_{\mathrm{ver}}^{\bullet}(E) \longrightarrow \mathfrak{X}^{\bullet}\left(E_{p}\right)$ is a surjective homomorphism of Gerstenhaber algebras, i.e. for all $X, Y \in \mathfrak{X}_{\mathrm{ver}}^{\bullet}(E)$ we have

$$
\iota_{p}^{*}(X \wedge Y)=\iota_{p}^{*} X \wedge \iota_{p}^{*} Y \quad \text { and } \quad \iota_{p}^{*}(\llbracket X, Y \rrbracket)=\llbracket \iota_{p}^{*} X, \iota_{p}^{*} Y \rrbracket .
$$

iii.) The vertical multivector fields which are polynomial along the fibers are a Gerstenhaber subalgebra of $\mathfrak{X}_{\mathrm{ver}}^{\bullet}(E)$ isomorphic to the Gerstenhaber algebra $\bigoplus_{\ell=0}^{\infty} \Gamma^{\infty}\left(\mathrm{S}^{\ell} E^{*} \otimes \Lambda^{\bullet} E\right)$, equipped with its canonical fiberwise Gerstenhaber algebra structure.

Note that for $X, Y \in \Gamma^{\infty}\left(\Lambda^{\bullet} T E\right)$ we have

$$
\llbracket X^{\vee}, Y^{\vee} \rrbracket=0 .
$$

\section{A.2 The vertical Hochschild-Kostant-Rosenberg theorem}

Recall that $k$-vector fields $X \in \mathfrak{X}^{k}(E)$ can be viewed as totally antisymmetric first order $k$ differential operators by use of the Hochschild-Kostant-Rosenberg map (for short: HKR map)

$$
\left(\mathrm{U}^{(1)}(X)\right)\left(f_{1}, \ldots, f_{k}\right)=\frac{1}{k !}\left\langle X, \mathrm{~d} f_{1} \otimes \cdots \otimes \mathrm{d} f_{k}\right\rangle,
$$

where $f_{1}, \ldots, f_{k} \in C^{\infty}(E)$. We denote by $\operatorname{HC}_{\text {diff }}^{k}\left(C^{\infty}(E)\right)$ the differential Hochschild $k$-cochains with values in $C^{\infty}(E)$, i.e. the $k$-differential operators

$$
\phi: \underbrace{C^{\infty}(E) \times \cdots \times C^{\infty}(E)}_{k \text {-times }} \longrightarrow C^{\infty}(E)
$$

Then $\phi \in \mathrm{HC}_{\mathrm{diff}}^{k}\left(C^{\infty}(E)\right)$ is called vertical if

$$
\phi\left(f_{1}, \ldots, \pi^{*} u f_{i}, \ldots, f_{k}\right)=\pi^{*} u \phi\left(f_{1}, \ldots, f_{k}\right)
$$

for all $f_{1}, \ldots, f_{k} \in C^{\infty}(E), u \in C^{\infty}(M)$ and $i=1, \ldots, k$. We denote the vertical $k$-differential operators by $\mathrm{HC}_{\mathrm{diff}, \mathrm{ver}}^{k}\left(C^{\infty}(E)\right)$. Clearly, $\mathrm{U}^{(1)}(X) \in \mathrm{HC}_{\mathrm{diff,ver}}^{k}\left(C^{\infty}(E)\right)$ for a vertical $k$-vector field $X$. The restriction of $\mathrm{U}^{(1)}$ to vertical multivector fields is denoted by

$$
\mathrm{U}_{\mathrm{ver}}^{(1)}: \mathfrak{X}_{\mathrm{ver}}^{\bullet}(E) \longrightarrow \mathrm{HC}_{\mathrm{diff}, \mathrm{ver}}^{\bullet}\left(C^{\infty}(E)\right)
$$

Let $R=\left(r_{1}, \ldots, r_{k}\right) \in \mathbb{N}^{k}$ be the multi-order of $\phi \in \mathrm{HC}_{\text {diff,ver }}^{k}\left(C^{\infty}(E)\right)$. Then locally

$$
\left.\phi\left(f_{1}, \ldots, f_{k}\right)\right|_{\pi^{-1}(U)}=\sum_{L=0}^{R} \frac{1}{L !} \phi_{L}^{\alpha_{1}^{1} \cdots \alpha_{\ell_{1}}^{1} \cdots \alpha_{1}^{k} \cdots \alpha_{\ell_{k}}^{k}} \frac{\partial^{\ell_{1}} f_{1}}{\partial s^{\alpha_{1}^{1}} \cdots \partial s^{\alpha_{\ell_{1}}^{1}}} \cdots \frac{\partial^{\ell_{k}} f_{k}}{\partial s^{\alpha_{1}^{k}} \cdots \partial s^{\alpha_{\ell_{k}}^{k}}}
$$

with unique functions $\phi_{L}^{\alpha_{1}^{1} \cdots \alpha_{\ell_{1}}^{1} \cdots \alpha_{1}^{k} \cdots \alpha_{\ell_{k}}^{k}} \in C^{\infty}\left(\pi^{-1}(U)\right)$, totally symmetric in $\left(\alpha_{1}^{i}, \ldots, \alpha_{\ell_{i}}^{i}\right)$ for all $i=1, \ldots, k$. Conversely, if $\phi \in \mathrm{HC}_{\text {diff }}^{k}\left(C^{\infty}(E)\right)$ is locally of this form, then $\phi$ is vertical. In this case it is easy to see that for all $L \leq R$

$$
\left.\sigma_{L}(\phi)\right|_{\pi^{-1}(U)}=\frac{1}{L !} \phi_{L}^{\alpha_{1}^{1} \cdots \alpha_{\ell_{1}}^{1} \cdots \alpha_{1}^{k} \cdots \alpha_{\ell_{k}}^{k}} \frac{\partial}{\partial s^{\alpha_{1}^{1}}} \vee \cdots \vee \frac{\partial}{\partial s^{\alpha_{\ell_{1}}^{1}}} \otimes \cdots \otimes \frac{\partial}{\partial s^{\alpha_{1}^{k}}} \vee \cdots \vee \frac{\partial}{\partial s^{\alpha_{\ell_{k}}^{k}}}
$$

defines a global tensor field $\sigma_{L}(\phi) \in \Gamma^{\infty}\left(\mathrm{S}^{\ell_{1}} \operatorname{Ver}(E) \otimes \cdots \otimes \mathrm{S}^{\ell_{k}} \operatorname{Ver}(E)\right)$, the $L$-symbol of $\phi$. Note that in general only the leading symbol, i.e. for $L=R$, has an invariant geometric interpretation 
as a tensor field. Conversely, out of a given tensor field $A \in \Gamma^{\infty}\left(\mathrm{S}^{\ell_{1}} \operatorname{Ver}(E) \otimes \cdots \otimes \mathrm{S}^{\ell_{k}} \operatorname{Ver}(E)\right)$ one can build a unique vertical $k$-differential operator $Q_{L}(A)$ of multi-order $L$ in a canonical way, such that $\sigma_{L}\left(Q_{L}(A)\right)=A$ and $\sigma_{L^{\prime}}\left(Q_{L}(A)\right)=0$ for all $L^{\prime} \neq L$.

Vertical multidifferential operators can again be restricted to fibers and yield multidifferential operators on $E_{p}$ for each $p \in M$. We denote the restriction again by

$$
\iota_{p}^{*}: \mathrm{HC}_{\mathrm{diff}, \mathrm{ver}}^{\bullet}\left(C^{\infty}(E)\right) \longrightarrow \mathrm{HC}_{\mathrm{diff}}^{\bullet}\left(C^{\infty}\left(E_{p}\right)\right) .
$$

Vertical multidifferential operators behave well under multicomposition: if $\phi_{i} \in \operatorname{HC}_{\text {diff, ver }}^{\ell_{i}}\left(C^{\infty}(E)\right)$ for $i=1, \ldots, k$ and $\phi \in \operatorname{HC}_{\text {diff,ver }}^{k}\left(C^{\infty}(E)\right)$ are given then $\phi \circ\left(\phi_{1} \otimes \cdots \otimes \phi_{k}\right) \in \operatorname{HC}_{\text {diff,ver }}^{\ell_{1}+\cdots+\ell_{k}}\left(C^{\infty}(E)\right)$. This is obvious from the definition (A.8). Moreover, in this case we have

$$
\iota_{p}^{*}\left(\phi \circ\left(\phi_{1} \otimes \cdot \otimes \phi_{k}\right)\right)=\left(\iota_{p}^{*} \phi\right) \circ\left(\iota_{p}^{*} \phi_{1} \otimes \cdots \otimes \iota_{p}^{*} \phi_{k}\right) .
$$

From this we immediately have the following statement:

Proposition A.2 Let $\pi: E \longrightarrow M$ be a vector bundle and $p \in M$.

i.) The vertical differential Hochschild cochains $\mathrm{HC}_{\text {diff,ver }}^{\bullet}\left(C^{\infty}(E)\right)$ are closed under the cupproduct $\cup$, the Hochschild differential $\delta$, and the Gerstenhaber bracket $[\cdot, \cdot]$.

ii.) The restriction map

$$
\iota_{p}^{*}: \mathrm{HC}_{\mathrm{diff}, \mathrm{ver}}^{\bullet}\left(C^{\infty}(E)\right) \longrightarrow \mathrm{HC}_{\mathrm{diff}}^{\bullet}\left(C^{\infty}\left(E_{p}\right)\right)
$$

is compatible with the cup-products, the Hochschild differentials and the Gerstenhaber brackets, respectively.

Proof: See e.g. [17] for the definition of $\cup, \delta$, and $[\cdot, \cdot]$. The statement follows immediately from the compatibility with the multicomposition and (A.13).

In particular, $\mathrm{HC}_{\mathrm{diff}, \mathrm{ver}}^{\bullet}\left(C^{\infty}(E)\right)$ is a subcomplex of the usual differential Hochschild complex of $C^{\infty}(E)$. Its cohomology, the vertical Hochschild cohomology of $C^{\infty}(E)$, will be denoted by $\mathrm{HH}_{\text {diff,ver }}^{\bullet}\left(C^{\infty}(E)\right)$. It is well-known that the cup-product and the Gerstenhaber bracket pass to the differential Hochschild cohomology $\mathrm{HH}_{\text {diff }}^{\bullet}\left(C^{\infty}(E)\right)$ which then becomes a Gerstenhaber algebra. To show the appropriate algebraic identities between $\cup$ and $[\cdot, \cdot]$ one has to construct explicit coboundaries, see [17]. From these explicit formulas it can easily be seen that the relevant coboundaries can be chosen vertical if all involved cocycles are vertical. Hence one has the following result:

Proposition A.3 Let $\pi: E \longrightarrow M$ be a vector bundle and $p \in M$.

i.) The vertical differential Hochschild cohomology of $C^{\infty}(E)$ becomes a Gerstenhaber algebra with respect to the cup-product and the Gerstenhaber bracket. The canonical map

$$
\mathrm{HH}_{\text {diff,ver }}^{\bullet}\left(C^{\infty}(E)\right) \longrightarrow \mathrm{HH}_{\mathrm{diff}}^{\bullet}\left(C^{\infty}(E)\right)
$$

is a map of Gerstenhaber algebras.

ii.) The restriction map $\iota_{p}^{*}$ induces a map of Gerstenhaber algebras

$$
\iota_{p}^{*}: \mathrm{HH}_{\mathrm{diff}, \text { ver }}^{\bullet}\left(C^{\infty}(E)\right) \longrightarrow \mathrm{HH}_{\mathrm{diff}}^{\bullet}\left(C^{\infty}\left(E_{p}\right)\right) .
$$


Let us now compute $\mathrm{HH}_{\text {diff, ver }}^{\bullet}\left(C^{\infty}(E)\right)$. We start with the trivial vector bundle $E=U \times \mathbb{R}^{d}$. In this case we consider $C^{\infty}\left(U \times \mathbb{R}^{d}\right)$ as a symmetric $C^{\infty}\left(\mathbb{R}^{d}\right)$-bimodule in the canonical way. If $\phi \in \mathrm{HC}_{\text {diff }}^{k}\left(C^{\infty}\left(\mathbb{R}^{d}\right), C^{\infty}\left(U \times \mathbb{R}^{d}\right)\right)$ is a $k$-differential operator on $C^{\infty}\left(\mathbb{R}^{d}\right)$ with values in $C^{\infty}\left(U \times \mathbb{R}^{d}\right)$ we can view this as a vertical $k$-differential operator $\widetilde{\phi} \in \mathrm{HC}_{\mathrm{diff,ver}}^{k}\left(C^{\infty}\left(U \times \mathbb{R}^{d}\right)\right)$ by setting

$$
\left(\widetilde{\phi}\left(f_{1}, \ldots, f_{k}\right)\right)(u, v)=\left(\phi\left(f_{1}(u, \cdot), \ldots, f_{k}(u, \cdot)\right)\right)(u, v) .
$$

Conversely, let $\Phi \in \mathrm{HC}_{\text {diff,ver }}^{k}\left(C^{\infty}\left(U \times \mathbb{R}^{d}\right)\right)$ then we can simply restrict $\Phi$ to the subalgebra $C^{\infty}\left(\mathbb{R}^{d}\right)$ of $C^{\infty}\left(U \times \mathbb{R}^{d}\right)$ and obtain $\widehat{\Phi} \in \mathrm{HC}_{\mathrm{diff}}^{k}\left(C^{\infty}\left(\mathbb{R}^{d}\right), C^{\infty}\left(U \times \mathbb{R}^{d}\right)\right)$. The following is obvious:

Lemma A.4 The extension $\phi \mapsto \widetilde{\phi}$ and the restriction $\Phi \mapsto \widehat{\Phi}$ are mutually inverse isomorphisms of Hochschild complexes

$$
\mathrm{HC}_{\text {diff }}^{k}\left(C^{\infty}\left(\mathbb{R}^{d}\right), C^{\infty}\left(U \times \mathbb{R}^{d}\right)\right) \cong \operatorname{HC}_{\text {diff,ver }}^{k}\left(C^{\infty}\left(U \times \mathbb{R}^{d}\right)\right) .
$$

Since $C^{\infty}\left(U \times \mathbb{R}^{d}\right)$ is a symmetric $C^{\infty}\left(\mathbb{R}^{d}\right)$-bimodule, we easily can compute its differential Hochschild cohomology using the Koszul 'resolution' as in [6]:

Lemma A.5 The HKR map induces an isomorphism

$\mathfrak{X}_{\mathrm{ver}}^{\bullet}\left(U \times \mathbb{R}^{d}\right) \cong \Lambda^{\bullet} \mathbb{R}^{d} \otimes C^{\infty}\left(U \times \mathbb{R}^{d}\right) \longrightarrow \mathrm{HH}_{\mathrm{diff}}^{\bullet}\left(C^{\infty}\left(\mathbb{R}^{d}\right), C^{\infty}\left(U \times \mathbb{R}^{d}\right)\right) \cong \operatorname{HH}_{\text {diff, ver }}\left(C^{\infty}\left(U \times \mathbb{R}^{d}\right)\right)$.

In particular, if $\phi \in \mathrm{HC}_{\mathrm{diff}, \mathrm{ver}}^{\bullet}\left(C^{\infty}\left(U \times \mathbb{R}^{d}\right)\right)$ is a cocycle then

$$
\phi=\mathrm{U}^{(1)}(X)+\delta \psi
$$

for some $\psi \in \mathrm{HC}_{\mathrm{diff,ver}}^{\bullet-1}\left(C^{\infty}\left(U \times \mathbb{R}^{d}\right)\right)$ and a unique $X \in \mathfrak{X}_{\mathrm{ver}}^{\bullet}\left(C^{\infty}\left(U \times \mathbb{R}^{d}\right)\right)$, given by the total antisymmetrization of $\phi$.

From this local statement the standard globalization argument as e.g. in [10] using a suitable partition of unity of $M$ and local trivializations of $E$ gives the following statement:

Lemma A.6 If $\phi \in \mathrm{HC}_{\mathrm{diff}, \text { ver }}^{\bullet}\left(C^{\infty}(E)\right)$ is a cocycle then there exists a unique vertical multivector field $X \in \mathfrak{X}_{\mathrm{ver}}^{\bullet}(E)$ and a vertical $\psi \in \mathrm{HC}_{\mathrm{diff} \text {,ver }}^{\bullet-1}\left(C^{\infty}(E)\right)$ such that

$$
\phi=\mathrm{U}_{\mathrm{ver}}^{(1)}(X)+\delta \psi
$$

and $X$ is obtained from the total antisymmetrization of $\phi$. In particular,

$$
\mathrm{U}_{\text {ver }}^{(1)}: \mathfrak{X}_{\text {ver }}^{\bullet}(E) \longrightarrow \mathrm{HH}_{\text {diff, ver }}^{\bullet}\left(C^{\infty}(E)\right)
$$

is an isomorphism.

Finally, $\mathrm{U}_{\text {ver }}^{(1)}$ as in (A.22) is not only an isomorphism of vector spaces but compatible with the Gerstenhaber algebra structures: First it is clear that on the level of cochains $U_{\text {ver }}^{(1)}$ maps $\wedge$-products to the total antisymmetrization of the corresponding $\cup$-products. Passing to cohomology, $\cup$ becomes supercommutative whence $\mathrm{U}_{\text {ver }}^{(1)}$ maps $\wedge$-products to $\cup$-products in cohomology. Moreover, $\mathrm{U}_{\text {ver }}^{(1)}$ is easily verified to map Schouten brackets of functions and vector fields to the corresponding Gerstenhaber brackets already on the level of cochains. Since functions and vector fields generate $\mathfrak{X}_{\text {ver }}^{\bullet}(E)$ by $\wedge$-products and since $\llbracket \cdot, \cdot \rrbracket$ as well as $[\cdot, \cdot]$ satisfy the same Leibniz rule (the latter only in cohomology) and since $U_{\text {ver }}^{(1)}$ is an isomorphism of associative supercommutative algebras, it follows that $\mathrm{U}_{\text {ver }}^{(1)}$ also maps Schouten brackets to Gerstenhaber brackets in cohomology. Note that on the level of cochains this is not true for higher multivector fields. We summarize the result of this section: 
Theorem A.7 (Vertical Hochschild-Kostant-Rosenberg Theorem) Let $\pi: E \longrightarrow M$ be a vector bundle and $p \in M$.

i.) The vertical HKR map gives an isomorphism of Gerstenhaber algebras

$$
\mathrm{U}_{\mathrm{ver}}^{(1)}: \mathfrak{X}_{\mathrm{ver}}^{\bullet}(E) \longrightarrow \mathrm{HH}_{\mathrm{diff}, \mathrm{ver}}^{\bullet}\left(C^{\infty}(E)\right) .
$$

ii.) Let $\mathrm{U}_{p}^{(1)}: \mathfrak{X}^{\bullet}\left(E_{p}\right) \longrightarrow \mathrm{HH}_{\mathrm{diff}}^{\bullet}\left(C^{\infty}\left(E_{p}\right)\right)$ be the usual HKR map on the vector space $E_{p}$. Then

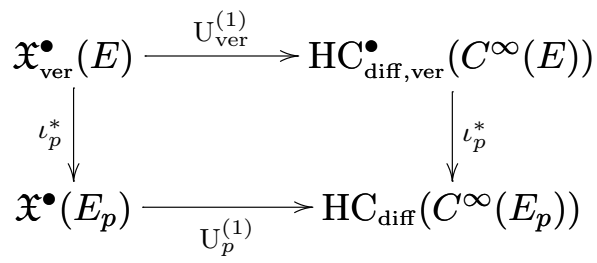

commutes and all maps are homomorphisms of Gerstenhaber algebras.

\section{A.3 The vertical formality}

We come now to the main theorem of this appendix, for which we shall recall some basic notions of formal deformation theory, see e.g. [11] or [6, Appendix], and the language of coalgebras, see e.g. [25, 29]. Let $\mathfrak{g}=\bigoplus_{k \in \mathbb{Z}} \mathfrak{g}_{k}$ be a differential graded Lie algebra with Lie bracket $[\cdot, \cdot]$ and differential $\delta$ of degree +1 . This structure can alternatively be described as follows. We denote the same vector space with shifted degree by +1 by $\mathfrak{g}[1]$ and consider the graded symmetric algebra $\mathrm{S}(\mathfrak{g}[1])$. With the graded symmetric tensor product $\vee$ and the graded cocommutative shuffle coproduct $\Delta_{\mathrm{sh}}$ one obtains a bialgebra $\mathrm{S}(\mathfrak{g}[1])$ with unit $\mathbb{1}$ and counit $\epsilon$ being just the projection on the tensor degree 0 . As coalgebra, $\mathrm{S}(\mathfrak{g}[1])$ is cofree within the category of augmented graded cocommutative counital coalgebras $\mathcal{C C}_{A N}$ with nilpotent augmentation ideal ker $\epsilon$, where a coalgebra is called augmented if there is exactly one group-like element denoted by $\mathbb{1}$. Note that $\mathrm{S}(\mathfrak{g}[1])$ is not cofree within the category of all graded cocommutative coalgebras, see [6,25,29] for further details. The differential $\delta$ and the bracket $[\cdot, \cdot]$ can be combined to a single map $\mathrm{d}=\delta+[\cdot, \cdot]: \mathrm{S}(\mathfrak{g}[1]) \longrightarrow \mathfrak{g}[1]$ of degree +1 . Since $\mathrm{S}(\mathfrak{g}[1])$ is in $\mathcal{C}_{A N}$ and thanks to the cofreeness, this map extends uniquely to a coderivation $\underline{\mathrm{d}}: \mathrm{S}(\mathfrak{g}[1]) \longrightarrow \mathrm{S}(\mathfrak{g}[1])$ such that $\operatorname{pr}_{\mathfrak{g}[1]} \stackrel{\circ}{\mathrm{d}}=\mathrm{d}$. Then $\delta^{2}=0$, the compatibility between $\delta$ and $[\cdot, \cdot]$ and the Jacobi identity for $[\cdot, \cdot]$ are all encoded in $\underline{\mathrm{d}}^{2}=0$.

Generalizing this gives the definition of an $L_{\infty}$-algebra (or Lie algebra up to homotopy): a graded vector space $\mathfrak{g}$ is called $L_{\infty}$-algebra if $\mathrm{S}(\mathfrak{g}[1])$ is equipped with a coderivation $\underline{\mathrm{d}}$ of degree +1 and $\underline{\mathrm{d}}^{2}=0$. If $\mathfrak{g}$ and $\mathfrak{h}$ are $L_{\infty}$-algebras then an $L_{\infty}$-morphism is a coalgebra morphism $\underline{\mathrm{U}}: \mathrm{S}(\mathfrak{g}[1]) \longrightarrow \mathrm{S}(\mathfrak{h}[1])$ such that $\underline{\mathrm{U}}^{\circ} \underline{\mathrm{d}}_{\mathfrak{g}}=\underline{\mathrm{d}}_{\mathfrak{h}} \circ \underline{\mathrm{U}}$. Clearly, in the above example of a differential graded Lie algebra, any morphism of differential graded Lie algebras induces an $L_{\infty}$-morphism. However, in general, there are more general $L_{\infty}$-morphisms than these: this is the key idea of Kontsevich's formality theorem.

If $\mathfrak{g}$ has an $L_{\infty}$-structure then the coderivation $\underline{\mathrm{d}}$ is uniquely determined by $\mathrm{d}=\mathrm{pr}_{\mathfrak{g}[1]} \circ \underline{\mathrm{d}}$. Similarly, an $L_{\infty}$-morphism $\underline{\mathrm{U}}$ is determined by $\mathrm{U}=\operatorname{pr}_{\mathfrak{h}[\mathbf{1}]} \circ \underline{U}$. Finally, each $d$ and $\mathrm{U}$ are determined by their Taylor coefficients

$$
\mathrm{d}=\sum_{n=1}^{\infty} \mathrm{d}^{(n)} \quad \text { and } \quad \mathrm{U}=\sum_{n=0}^{\infty} \mathrm{U}^{(n)},
$$

where $\mathrm{d}^{(n)}: \mathrm{S}^{n}(\mathfrak{g}[1]) \longrightarrow \mathfrak{g}[1]$ and $\mathrm{U}^{(n)}: \mathrm{S}^{n}(\mathfrak{g}[1]) \longrightarrow \mathfrak{h}[1]$, respectively. Necessarily, $\mathrm{U}^{(0)}$ maps $\mathbb{1}_{\mathrm{S}(\mathfrak{g}[1])}$ to $\mathbb{1}_{\mathrm{S}(\mathfrak{h}[1])}$. 
In general, $\delta=\mathrm{d}^{(1)}: \mathfrak{g}[1] \longrightarrow \mathfrak{g}[1]$ satisfies $\delta^{2}=0$ and $[\cdot, \cdot]=\mathrm{d}^{(2)}$ defines a Lie bracket 'up to $\delta$-homotopy', i.e. $[\cdot, \cdot]$ induces a graded Lie bracket on the $\delta$-cohomology. This explains the name $L_{\infty}$-algebra. Moreover, an $L_{\infty}$-morphism induces a morphism of graded Lie algebras in cohomology. One calls $\underline{\mathrm{U}}$ an $L_{\infty}$-quasiisomorphism or formality if the induced map in cohomology is an isomorphism.

In [22] Kontsevich constructed an $L_{\infty}$-quasiisomorphism $\underline{\mathrm{U}}$ between $\mathfrak{g}=\mathfrak{X}^{\bullet}\left(\mathbb{R}^{d}\right)[1]$ viewed as $L_{\infty}$-algebra with $\mathrm{d}=\mathrm{d}^{(2)}=\llbracket \cdot, \cdot \rrbracket$, and $\mathfrak{h}=\mathrm{HC}_{\text {diff }}^{\bullet}\left(C^{\infty}\left(\overline{\mathbb{R}^{d}}\right)\right)[1]$ viewed as $L_{\infty}$-algebra with $\mathrm{d}=\mathrm{d}^{(1)}+\mathrm{d}^{(2)}=\delta+[\cdot, \cdot]$. We recall the basic properties of this formality map:

Theorem A.8 (Kontsevich's Formality for $\mathbb{R}^{d}$ ) There exists an $L_{\infty}$-quasiisomorphism

$$
\underline{\mathrm{U}}_{\mathbb{R}^{d}}: \mathrm{S}\left(\mathfrak{X}^{\bullet}\left(\mathbb{R}^{d}\right)[2]\right) \longrightarrow \mathrm{S}\left(\mathrm{HC}_{\mathrm{diff}}^{\bullet}\left(C^{\infty}\left(\mathbb{R}^{d}\right)\right)[2]\right),
$$

such that the Taylor coefficients $\mathrm{U}_{\mathbb{R}^{d}}^{(n)}$ have the following properties:

i.) $\mathrm{U}_{\mathbb{R}^{d}}^{(1)}$ is the HKR map.

ii.) $\mathrm{U}_{\mathbb{R}^{d}}^{(n)}$ is a real $n$-differential operator on its $n$ arguments in $\mathfrak{X}^{\bullet}\left(\mathbb{R}^{d}\right)$ with constant coefficients.

iii.) $\mathrm{U}_{\mathbb{R}^{d}}^{(n)}$ is $\mathrm{GL}(d, \mathbb{R})$-invariant in the sense that for $\left.X_{1}, \ldots, X_{n} \in \mathfrak{X} \bullet \mathbb{R}^{d}\right), f_{1}, \ldots, f_{m} \in C^{\infty}\left(\mathbb{R}^{d}\right)$, and $A \in \mathrm{GL}(d, \mathbb{R})$ one has

$$
A^{*}\left(\left(\mathrm{U}_{\mathbb{R}^{d}}^{(n)}\left(X_{1}, \ldots, X_{n}\right)\right)\left(f_{1}, \ldots, f_{m}\right)\right)=\left(\mathrm{U}_{\mathbb{R}^{d}}^{(n)}\left(A^{*} X_{1}, \ldots, A^{*} X_{n}\right)\right)\left(A^{*} f_{1}, \ldots, A^{*} f_{m}\right),
$$

where $A$ acts by $x \mapsto A x$ as usual.

The important point for us is the $\mathrm{GL}(d, \mathbb{R})$-invariance. Using a local frame $e_{1}, \ldots, e_{d} \in$ $\Gamma^{\infty}\left(\left.E\right|_{U}\right)$, on a local trivialization $\left.E\right|_{U} \cong U \times \mathbb{R}^{d}$ we can define

$$
\left.\mathrm{U}_{\text {ver }}^{(n)}\left(X_{1}, \ldots, X_{n}\right)\right|_{E_{p}}=\mathrm{U}_{\mathbb{R}^{d}}^{(n)}\left(X_{1}(u, \cdot), \ldots, X_{n}(u, \cdot)\right),
$$

for $X_{1}, \ldots, X_{n} \in \mathfrak{X}_{\mathrm{ver}}^{\bullet}(E)$ and $p \in U$. We only use the linear coordinates $\left(s^{1}, \ldots, s^{d}\right)$ on $\left.E\right|_{U}$ and apply $\mathrm{U}_{\mathbb{R}^{d}}^{(n)}$ with respect to those, treating the $U$-directions as parameters not affected by $\mathrm{U}_{\mathbb{R}^{d}}^{(n)}$. Then from (A.27) it follows immediately, that for vertical $X_{1}, \ldots, X_{n}$ the operator $\mathrm{U}_{\text {ver }}^{(n)}\left(X_{1}, \ldots, X_{n}\right)$ is actually defined globally and independent on the choice of the trivialization. From this we obtain immediately the main result of this appendix, the vertical formality theorem:

Theorem A.9 (Vertical formality theorem) Let $\pi: E \longrightarrow M$ be a vector bundle. Then there exists a unique $L_{\infty}$-quasiisomorphism

$$
\underline{\mathrm{U}}_{\mathrm{ver}}: \mathrm{S}\left(\mathfrak{X}_{\mathrm{ver}}^{\bullet}(E)[2]\right) \longrightarrow \mathrm{S}\left(\mathrm{HC}_{\mathrm{diff,ver}}^{\bullet}\left(C^{\infty}(E)\right)[2]\right)
$$

which has the following properties:

i.) The Taylor coefficients $\mathrm{U}_{\text {ver }}^{(n)}$ are real vertical $n$-differential operators on their $n$ arguments with constant coefficients.

ii.) $\mathrm{U}_{\mathrm{ver}}^{(1)}$ is the HKR map. 
iii.) $\underline{\mathrm{U}}_{\mathrm{ver}}$ restricts to an $L_{\infty}$-quasiisomorphism $\underline{\mathrm{U}}_{p}$

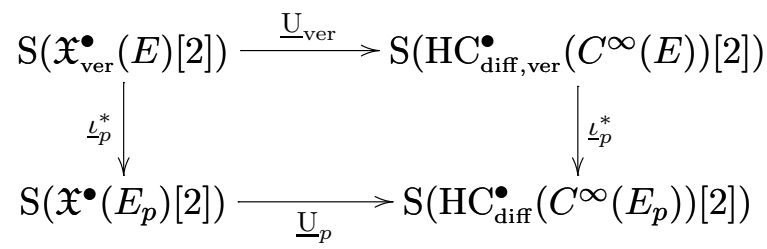

such that $\underline{\mathrm{U}}_{p}$ coincides with Kontsevich's formality on the vector space $E_{p}$ for all $p \in M$. Here $\underline{\iota}_{p}^{*}$ is the canonical extension of the restriction $\iota_{p}^{*}$ to a coalgebra morphism.

Proof: If $\underline{\mathrm{U}}_{\mathrm{ver}}$ is a vertical $L_{\infty}$-morphism like in (A.29) which satisfies the first part, then it clearly restricts to a $L_{\infty}$-morphism $\underline{\mathrm{U}}_{p}$ for all $p \in M$ such that (A.30) commutes. Moreover, such a $\underline{\mathrm{U}}_{\mathrm{ver}}$ is completely determined by its restrictions $\underline{\mathrm{U}}_{p}$ which proves uniqueness. For existence, we see that $\underline{\mathrm{U}}_{\mathrm{ver}}$ constructed above is an $L_{\infty}$-morphism since this can be checked locally whence we can rely on Theorem A.8 Moreover, the first and third part are clearly satisfied by construction and the second part follows from Theorem A.8 as well as Theorem A.7. Since by Theorem A.7 the vertical HKR map induces an isomorphism in cohomology, $\underline{\mathrm{U}}_{\mathrm{ver}}$ is a quasiisomorphism since this is always decided by the first Taylor coefficient.

\section{A.4 Vertical Poisson structures and vertical star products}

A vertical Poisson structure $\theta$ is a vertical bivector field $\theta \in \mathfrak{X}_{\mathrm{ver}}^{2}(E)$ with $\llbracket \theta, \theta \rrbracket=0$. Analogously, one defines a formal vertical Poisson structure $\theta=\sum_{r=0}^{\infty} \lambda^{r} \theta_{r} \in \mathfrak{X}_{\mathrm{ver}}^{2}(E)[[\lambda]]$. Two formal vertical Poisson structures $\theta, \theta^{\prime}$ are called vertically equivalent if there exists a formal vertical vector field $X \in \mathfrak{X}_{\mathrm{ver}}^{1}(E)[[\lambda]]$ such that

$$
\theta^{\prime}=\mathrm{e}^{\lambda \mathscr{L}_{X}}(\theta) .
$$

One calls $\mathrm{e}^{\lambda \mathscr{L}_{X}}$ also a formal diffeomorphism. Note that in this case the zeroth order parts of $\theta$ and $\theta^{\prime}$ coincide $\theta_{0}=\theta_{0}^{\prime}$.

If $\theta$ is a vertical Poisson structure then $\theta_{p}=\iota_{p}^{*} \theta \in \mathfrak{X}^{2}\left(E_{p}\right)$ is a Poisson structure on the vector space $E_{p}$. The map $\iota_{p}^{*}: C^{\infty}(E) \longrightarrow C^{\infty}\left(E_{p}\right)$ then becomes a Poisson map. Clearly, vertically equivalent $\theta, \theta^{\prime} \in \mathfrak{X}_{\text {ver }}^{2}(E)[[\lambda]]$ restrict to equivalent $\theta_{p}, \theta_{p}^{\prime} \in \mathfrak{X}^{2}\left(E_{p}\right)[[\lambda]]$ via the restriction $X_{p}=\iota_{p}^{*} X$ of $X$.

A Poisson structure $\theta \in \mathfrak{X}^{2}(E)$ induces a Poisson bracket $\{\cdot, \cdot\}_{\theta}$ on $C^{\infty}(E)$ as usual by $\{f, g\}_{\theta}=$ $\langle\theta, \mathrm{d} f \otimes \mathrm{d} g\rangle$. Then $\theta$ is vertical iff $\left\{f, \pi^{*} u\right\}_{\theta}=0$ for all $f \in C^{\infty}(E)$ and $u \in C^{\infty}(M)$, i.e. $\pi^{*} C^{\infty}(M) \subseteq C^{\infty}(E)$ is part of the Poisson center of $\{\cdot, \cdot\}_{\theta}$.

Example A.10 Let $X \in \Gamma^{\infty}\left(\Lambda^{2} E\right)$ be an arbitrary section. Then the vertical lift $\theta=X^{\vee}$ is a vertical Poisson structure by (A.5). Clearly, $\theta_{p}$ is a constant Poisson structure on $E_{p}$.

Example A.11 Let $E_{p}$ be equipped with a Lie algebra structure $[\cdot, \cdot]_{p}$ depending smoothly on $p \in M$. Then on $E^{*}$ we have a linear vertical Poisson structure in the usual way, which restricts to the canonical linear Poisson structure on each $E_{p}^{*}$ induced by the Lie bracket $[\cdot, \cdot]_{p}$. More generally, one can consider quadratic and higher order vertical Poisson structures.

Example A.12 Consider first the local situation $\left.E\right|_{U} \cong U \times \mathbb{R}^{d}$. Then we can choose $d$ commuting vector fields $X_{1}, \ldots, X_{d}$ on $\mathbb{R}^{d}$ whose supports are contained in a small ball $B_{\epsilon}(0)$ around 0 and such that $X_{\alpha}(0)=e_{\alpha}$ for all $\alpha=1, \ldots, 0$. It is well-known that such vector fields exist. Moreover, 
let $\Theta \in \Gamma^{\infty}\left(\left.\Lambda^{2} E\right|_{U}\right)$ be an arbitrary section of $\left.\Lambda^{2} E\right|_{U}$, locally written as $\Theta=\frac{1}{2} \Theta^{\alpha \beta} e_{\alpha} \wedge e_{\beta}$. Then $\theta=\frac{1}{2} \pi^{*} \Theta^{\alpha \beta} X_{\alpha} \wedge X_{\beta} \in \mathfrak{X}_{\mathrm{ver}}^{2}\left(\left.E\right|_{U}\right)$ is a Poisson structure such that $\theta_{p}$ has compact support around $0_{p} \in E_{p}$ and $\theta\left(0_{p}\right)=\Theta^{\vee}\left(0_{p}\right)$ for all $p \in U$. Hence there are 'many' vertical Poisson structures with compactly supported $\theta_{p}$. In the global situation we still have many of them but it is not clear whether we also can arrange to get every vertical lift at the zero section.

Let us now turn to star products. A star product $\star=\sum_{r=0}^{\infty} \lambda^{r} C_{r}$ is called vertical if all $C_{r}$ are vertical bidifferential operators. In particular, the antisymmetric part of $C_{1}$ defines a vertical Poisson structure $\theta$ by $\{f, g\}_{\theta}=\frac{1}{\mathrm{i}}\left(C_{1}(f, g)-C_{1}(g, f)\right)$. In this case we say that $\star$ quantizes $\theta$.

A star product $\star$ is vertical iff

$$
f \star \pi^{*} u=f \pi^{*} u=\pi^{*} u \star f
$$

for all $f \in C^{\infty}(E)$ and $u \in C^{\infty}(M)$. Two vertical star products $\star$ and $\star^{\prime}$ are vertically equivalent is there exists a formal series $S=\mathrm{id}+\sum_{r=1}^{\infty} \lambda^{r} S_{r}$ of vertical differential operators $S_{r}$ such that

$$
S\left(f \star^{\prime} g\right)=S f \star S g \quad \text { and } \quad S 1=1 .
$$

Analogously to vertical Poisson structures we can also restrict vertical star products $\star$ to star products $\star_{p}$ for $C^{\infty}\left(E_{p}\right)[[\lambda]]$. Clearly, $\star_{p}$ is still associative by A.13 and we have

$$
\iota_{p}^{*}(f \star g)=\iota_{p}^{*} f \star_{p} \iota_{p}^{*} g .
$$

Moreover, vertically equivalent $\star$ and $\star^{\prime}$ restrict to equivalent $\star_{p}$ and $\star_{p}^{\prime}$.

Using the vertical formality theorem one immediately obtains the following existence and classification theorem by general arguments analogous to [22]:

Theorem A.13 (Vertical star products) Let $\pi: E \longrightarrow M$ be a vector bundle and $\underline{\mathrm{U}}_{\mathrm{ver}}$ the vertical formality from Theorem A.9.

i.) For a formal vertical Poisson structure $\theta \in \mathfrak{X}_{\mathrm{ver}}^{2}(E)[[\lambda]]$ the definition

$$
f \star_{\theta} g=f g+\sum_{r=1}^{\infty}\left(\frac{\mathrm{i} \lambda}{2}\right)^{r}\left(\mathrm{U}_{\text {ver }}^{(r)}(\theta, \ldots, \theta)\right)(f, g)
$$

yields a vertical star product $\star_{\theta}$ quantizing $\theta_{0}$. If $\theta=\bar{\theta}$ is real, $\star_{\theta}$ is Hermitean.

ii.) The map $\theta \mapsto \star_{\theta}$ induces a bijection on the level of vertical equivalence classes of formal vertical Poisson structures and vertical star products, respectively.

iii.) The restriction $\left(\star_{\theta}\right)_{p}$ coincides with $\star_{\theta_{p}}$ which is the Kontsevich star product corresponding to $\theta_{p}$ on the vector space $E_{p}$ for $p \in M$.

\section{References}

[1] Bahns, D.: Local Counterterms on the Noncommutative Minkowski space. In: Boutet DE Monvel, A., Buchholz, D., Iagolnitzer, D., Moschella, U. (eds.): Rigorous Quantum Field Theory. A Festschrift for Jacques Bros, vol. 251 in Progress in Mathematics. Birkhäuser, 2006.

[2] Bahns, D., Doplicher, S., Fredenhagen, K., Piacitelli, G.: Ultraviolet finite quantum field theory on quantum spacetime. Commun. Math. Phys. 237 (2003), 221-241.

[3] Bayen, F., Flato, M., Frønsdal, C., Lichnerowicz, A., Sternheimer, D.: Deformation Theory and Quantization. Ann. Phys. 111 (1978), 61-151. 
[4] Bieliavsky, P.: Strict Quantization of Solvable Symmetric Spaces. J. of Symplectic Geometry 1.2 (2002), 269-320.

[5] Borchers, H.-J., Pohlmeyer, K.: Eine scheinbare Abschwächung der Lokalitätsbedingung II. Commun. Math. Phys. 8 (1968), 269-281.

[6] Bordemann, M., Ginot, G., Halbout, G., Herbig, H.-C., Waldmann, S.: Formalité Go adaptee et starreprésentations sur des sous-variétés coïsotropes. Preprint math.QA/0504276 (2005), 56 pages. Extended version of the previous preprint math/0309321

[7] Bursztyn, H., Waldmann, S.: Deformation Quantization of Hermitian Vector Bundles. Lett. Math. Phys. 53 (2000), 349-365.

[8] Bursztyn, H., Waldmann, S.: On Positive Deformations of ${ }^{*}$-Algebras. In: Dito, G., Sternheimer, D. (EDS.): Conférence Moshé Flato 1999. Quantization, Deformations, and Symmetries, Mathematical Physics Studies no. 22, 69-80. Kluwer Academic Publishers, Dordrecht, Boston, London, 2000.

[9] Bursztyn, H., Waldmann, S.: Hermitian star products are completely positive deformations. Lett. Math. Phys. 72 (2005), 143-152.

[10] Cahen, M., Gutt, S., DeWilde, M.: Local Cohomology of the Algebra of C ${ }^{\infty}$ Functions on a Connected Manifold. Lett. Math. Phys. 4 (1980), 157-167.

[11] Cattaneo, A. S.: Formality and Star Products. In: Gutt, S., Rawnsley, J., Sternheimer, D. (eds.): Poisson Geometry, Deformation Quantisation and Group Representations, vol. 323 in London Mathematical Society Lecture Note Series, 79-144. Cambridge University Press, Cambridge, 2005. Lecture Notes taken by D. Indelicato.

[12] Cattaneo, A. S., Felder, G., Tomassini, L.: From local to global deformation quantization of Poisson manifolds. Duke Math. J. 115.2 (2002), 329-352.

[13] Dito, G., Sternheimer, D.: Deformation quantization: genesis, developments and metamorphoses. In: Halbout, G. (EDS.): Deformation quantization, vol. 1 in IRMA Lectures in Mathematics and Theoretical Physics, 9-54. Walter de Gruyter, Berlin, New York, 2002.

[14] Dolgushev, V. A.: Covariant and equivariant formality theorems. Adv. Math. 191 (2005), 147-177.

[15] Doplicher, S., Fredenhagen, K., Roberts, J. E.: The Quantum Structure of Spacetime at the Planck Scale and Quantum Fields. Commun. Math. Phys. 172 (1995), 187-220.

[16] Fedosov, B. V.: Deformation Quantization and Index Theory. Akademie Verlag, Berlin, 1996.

[17] Gerstenhaber, M.: Cohomology Structure of an associative Ring. Ann. Math. 78 (1963), 267-288.

[18] Gutt, S.: Variations on deformation quantization. In: Dito, G., Sternheimer, D. (Eds.): Conférence Moshé Flato 1999. Quantization, Deformations, and Symmetries, Mathematical Physics Studies no. 21, 217254. Kluwer Academic Publishers, Dordrecht, Boston, London, 2000.

[19] Heller, J. G., Neumaier, N., Waldmann, S.: A $C^{*}$-Algebraic Model for Locally Noncommutative SpaceTimes. Preprint, work in progress (2006).

[20] Jurčo, B., Möller, L., Schraml, S., Schupp, P., Wess, J.: Construction of non-Abelian gauge theories on noncommutative spaces. Eur. Phys. J. C21 (2001), 383-388.

[21] JurČO, B., Schupp, P., Wess, J.: Noncommutative gauge theory for Poisson manifolds. Nucl. Phys. B584 (2000), 784-794.

[22] Kontsevich, M.: Deformation Quantization of Poisson manifolds. Lett. Math. Phys. 66 (2003), $157-216$.

[23] Landsman, N. P.: Mathematical Topics between Classical and Quantum Mechanics. Springer Monographs in Mathematics. Springer-Verlag, Berlin, Heidelberg, New York, 1998.

[24] Liao, Y., Sibold, K.: Spectral representation and dispersion relations in field theory on noncommutative space. Phys. Lett. B 549 (2002), 352-361.

[25] Markl, M., Shnider, S., Stasheff, J.: Operads in Algebra, Topology and Physics. American Mathematical Society, Providence, Rhode Island, 2002.

[26] Pohlmeyer, K.: Eine scheinbare Abschwächung der Lokalitätsbedingung. Commun. Math. Phys. 7 (1968), 80-92.

[27] Rieffel, M. A.: Deformation quantization for actions of $\mathbb{R}^{d}$. Mem. Amer. Math. Soc. 106.506 (1993), 93 pages. 
[28] RIEFFEL, M. A.: On the operator algebra for the space-time uncertainty relations. In: DopLICHER, S., LONGO, R., Roberts, J. E., Zsido, L. (EDS.): Operator algebras and quantum field theory, 374-382. International Press, Cambridge, MA, 1997. Proceedings of the conference held in Rome, July 1-6, 1996.

[29] Sweedler, M.: Hopf Algebras. W. A. Benjamin, Inc, New York, 1969.

[30] Waldmann, S.: Deformation of Hermitian Vector Bundles and Non-Commutative Field Theory. In: MaEDA, Y., Watamura, S. (eds.): Noncommutative Geometry and String Theory, vol. 144 in Prog. Theo. Phys. Suppl., 167-175. Yukawa Institute for Theoretical Physics, 2001. Proceedings of the International Workshop on Noncommutative Geometry and String Theory.

[31] Waldmann, S.: Morita Equivalence, Picard Groupoids and Noncommutative Field Theories. In: CarowWatamura, U., Maeda, Y., Watamura, S. (eds.): Quantum Field Theory and Noncommutative Geometry, vol. 662 in Lect. Notes Phys., 143-155. Springer-Verlag, Berlin, Heidelberg, 2005.

[32] Waldmann, S.: States and Representation Theory in Deformation Quantization. Rev. Math. Phys. 17 (2005), $15-75$.

[33] Waldmann, S.: Poisson-Geometrie und Deformationsquantisierung. Fakultät für Mathematik und Physik, Albert-Ludwigs-Universität, 2006. Lecture notes for the lecture 'Poisson-Geometrie und Deformationsquantisierung' held in Freiburg in 2003/2004, ca. 450 pages in german. Available at http://idefix.physik. uni-freiburg.de/ stefan/. 